\title{
A frequency domain method for scattering problems with moving boundaries
}

\author{
D. Gasperini* ${ }^{* \dagger}$ H. P. Beise*, U. Schroeder*, X. Antoine ${ }^{\dagger}$, C. Geuzaine ${ }^{\ddagger}$
}

\begin{abstract}
We propose a multi-harmonic numerical method for solving wave scattering problems with moving boundaries, where the scatterer is assumed to move smoothly around an equilibrium position. We first develop an analysis to justify the method and its validity in the one-dimensional case with small-amplitude sinusoidal motions of the scatterer, before extending it to large-amplitude, arbitrary motions in oneand two-dimensional settings. We compare the numerical results of the proposed approach to standard space-time resolution schemes, which illustrates the efficiency of the new method.
\end{abstract}

Keywords: high frequency scattering; scalar waves; moving boundary; Doppler effect; multifrequency resolution.

\section{Introduction}

In wave scattering theory, it is well-known that the motion of the target modulates the frequency of the reflected wave, which is the so-called Doppler effect [15, 30]. For a motion with uniform velocity, the Doppler frequency shift can be computed easily [13]. The prediction of Doppler shifts for more general movements is commonly obtained through simple approximate models combined with signal processing [9, 10, 15, 16, 31, 38, 40.

The radar detection of non uniformly moving scatterers has found a tremendous number of applications in recent years, thanks to the availability of new high-frequency sensors and devices. For example, the radar frequency ranges $24-24.5 \mathrm{GHz}$ and $61-61.5 \mathrm{GHz}$ (the socalled ISM bands) are standard for many applications, and the new $77-81 \mathrm{GHz}$ band is currently considered for automotive applications. The $\mathrm{THz}$ frequency range will appear in applications in the next few years, e.g. at $140 \mathrm{GHz}$. A major advantage of high-frequency radar sensing is its sensitivity to micro movements [15, 16], when the scatterer includes

\footnotetext{
${ }^{*}$ IEE S.A., Bissen, Luxembourg

†Université de Lorraine, CNRS, Inria, IECL, F-54000 Nancy, France

‡University of Liège, Montefiore Institute B28, B-4000 Liège, Belgium
} 
several moving parts. This results in the so-called micro-Doppler effect [15, 16, which is used with great success from drone detection [8] or the analysis of pedestrian movement [10, 16, 29] to the modeling of the effect of the rotation of helicopter rotor blades on the radar signature of the aircraft [2]. In the automotive industry, micro-Doppler sensing has recently been proposed [23, 31, 36, 38] for the contactless detection of vital signs like breathing of infants left alone on the back seat of overheating cars. The engineering of such very high frequency radar sensing devices entails dealing with multiple challenges, as for instance the analysis of random body movements and vehicle vibrations [23, 31, 38, 39, 40, 43, leading to radar signatures that can be classified for example by deep learning techniques [7, 8, 18]. In order to design these new sensors, an adequate full realistic simulation of the underlying high frequency scenarios is crucial.

Concretely, a suitable physical modeling of the (direct) problem leads to solve a timedependent wave propagation problem in a complex environment (e.g. the interior of a car), which has both a complex geometrical shape and involves various materials that interact strongly with the high frequency emitted signal. In addition, the moving target is also usually of complex shape and materials (e.g. the infant on the back seat of the car), and characterized by small amplitude displacements at extremely low frequencies compared to the emitter. The natural mathematical framework to model such physical problems is the derivation of an adapted system of partial differential equations (PDEs), which are to be solved numerically when the configuration under study involves complex geometries or materials. Additional effects such as random vibrations can be handled by adding or modifying the PDE system.

The solution in a PDE setting of moving target problems has already received some attention in the mathematical and engineering communities. Analytical approaches for solving wave-like problems with simple motions have been developed e.g. for rotating obstacles [12, 17, 21, 45] or vibrating objects [14, 17, 34, 40]. In addition, numerical schemes based e.g. on FDTD [34, 49, 50] or fast integral equation solvers [48] were also investigated. More mathematical works related essentially to one-dimensional moving boundary problems have also been proposed e.g. by Fokas and his co-authors [28] to recast the problem as a Volterra integral equation in a fixed domain, or by Christov and Christov [20] for an asymptotic multiscale analysis of the Doppler effect in a half-space. To the best of the authors' knowledge, however, the numerical solution of the micro-Doppler PDE modeling problem has not been addressed yet.

In the present paper, we propose an original frequency domain method to address this problem, which leads to the solution of coupled systems of Helmholtz-type equations. First, we transform the constant coefficients wave equation in the moving domain as a new wavelike equation in a fixed domain but with variable coefficients related to the metric change (a similar approach was used for quasi-static electromagnetic models in [33]). Since we are studying the micro-Doppler problem (small amplitude and low frequency oscillations of the scatterer) for a high frequency radar, we can then expand the solution in the Fourier domain in time as a Fourier series expansion centered around the radar frequency, modulated by the low frequency perturbation induced by the scatterer movement. For small amplitude movements, the variable coefficients wave equation can be simplified thanks to 
the small amplitude, and the series expansion can be truncated to keep a finite number of discrete frequency components related to the small amplitude variations. The resulting approach then yields a coupled system of Hemholtz equations for the wave numbers defined by the discrete frequency components kept by the approximation. For larger amplitude movements, a similar analysis can be developed based on adding more Fourier modes since the frequency coupling is stronger, resulting in a larger system of coupled Helmholtz equations with variable coefficients. This approach is valid for any dimension and configuration, and the resulting frequency domain coupled formulation can be solved by efficient numerical methods adapted to solving Helmholtz-type equations in the high frequency regime. In the present paper, we propose an approach based on the finite element method [25] which is known to be flexible to handle two- and three-dimensional complex engineering configurations, including complex materials and shapes. In addition, this choice allows to consider in the future an algorithmic adaptation of efficient high-order finite element solvers based on domain decomposition [4, 24, 27, 44, where only a local resolution of the problem around the moving obstacle could be resolved. Let us remark that, depending on the problem, other high frequency numerical methods may also be adapted like for example fast integral equations solvers [19] or even asymptotic adpproximation techniques [5]. Finally, let us notice that the finite Fourier expansion method which leads to the coupled system of PDEs has also been used in the past under the name of the harmonic-balance method or the multi-harmonic approach [6, 11, 32, 33, 35, 41, 46]. It has been proved to be particularly efficient for engineering problems, including situations related to wave-like equations [22, 26, 47].

The paper is organized as follows. In Section 2 we define a sine-motion moving boundary scattering problem and formulate it in a fixed domain. In Section 3 we develop a multiharmonic approach to solve the scattering problem for small and large amplitudes of the boundary motion. We illustrate in Section 4 the validity of the method through numerical computations and generalize it in Section 5 to general boundary motions which are not explicitly prescribed. In Section 6, we extend the approach to higher-dimensional domains and illustrate the numerical method on a two-dimensional example. Finally, we conclude in Section 7. Appendix A details some computations.

\section{Problem statement and reformulation in a fixed do- main}

\subsection{The initial boundary-value problem}

We assume that the bounded spatial domain is defined by: $\Omega(t):=] 0, \ell(t)[$, where $t \in \mathbb{R}$ is the time variable and $x$ denotes the spatial variable. The modeling of the moving boundary is described by the time-dependent function $\ell(t)$. In the context of this work, $\ell(t)$ is supposed to be smooth and bounded. For $\left.\mathbb{R}_{*}^{+}:=\right] 0, \infty[$, we introduce the unknown total wave field $u:=u(x, t)$, for $x \in \Omega(t)$ and $t \in \mathbb{R}_{*}^{+}$, solution to the constant coefficients 
wave equation

$$
\partial_{t t} u-c^{2} \partial_{x x} u=0
$$

where $c$ is the wave velocity in the medium under consideration. At the fixed left boundary $x=0$ of $\Omega(t)$, we impose a single-source term $A \sin \left(\omega_{\mathrm{f}} t\right)$, with amplitude $A \in \mathbb{R}_{*}^{+}$and angular frequency $\omega_{\mathrm{f}}:=2 \pi \nu_{\mathrm{f}}>0$. In practice, $\nu_{\mathrm{f}}$ is expected to be very large compared to the frequency content of $\ell(t)$. For instance, $\nu_{\mathrm{f}}$ might be in frequency range $10^{9}-10^{10}$ for realistic radar applications. The associated inhomogeneous Dirichlet boundary condition is then set as:

$$
u(0, t)=A \sin \left(\omega_{\mathrm{f}} t\right) .
$$

On the moving right boundary, we fix a homogeneous Dirichlet boundary condition

$$
u(\ell(t), t)=0 .
$$

For physical applications, we consider the time origin of the problem as an emission starting at $t=0$, and take into account the transient mode of the excited wave field. Finally, for the sake of well-posedness, we add the two initial conditions

$$
u(x, 0)=0
$$

and

$$
\partial_{t} u(x, 0)=0
$$

\subsection{The case of a motionless boundary}

Let us consider the case where the right boundary is fixed, i.e. $\ell(t):=L$. Then, we get an explicit form of the solution (see Appendix A for a proof).

Proposition 1. Let us assume that : $\Omega(t)=\Omega:=] 0, L\left[, \forall t>0\right.$. We define $\kappa_{\mathrm{f}}:=\omega_{\mathrm{f}} / c$, $\kappa_{m}:=m \pi / L$ and $\omega_{m}:=c \kappa_{m}$, for $m \in \mathbb{N}$. We suppose that: $\omega_{\mathrm{f}} \neq \omega_{m}, \forall m \in \mathbb{N}$. Then, the solution $u_{0}$ of (1) is given by: $\forall x \in \Omega, t>0$,

$$
u_{0}=u_{0, \nu_{\mathrm{f}}}+\sum_{m \in \mathbb{N}} u_{0, \nu_{m}}
$$

where

$$
\left\{\begin{array}{l}
u_{0, \nu_{\mathrm{f}}}(x, t):=\frac{A}{\sin \left(\kappa_{\mathrm{f}} L\right)} \sin \left(\kappa_{\mathrm{f}}(L-x)\right) \sin \left(\omega_{\mathrm{f}} t\right), \\
u_{0, \nu_{m}}(x, t):=\frac{2 A \kappa_{\mathrm{f}}}{L\left(\kappa_{\mathrm{f}}^{2}-\kappa_{m}^{2}\right)} \sin \left(\kappa_{m} x\right) \sin \left(\omega_{m} t\right) .
\end{array}\right.
$$

Let us remark that $u_{0}$ can be extended to $t \in \mathbb{R}$, if we consider the source term (2) emitting for $t<0$, and the conditions (3), (4) and (5). Hence, this allows us to introduce $\widehat{u}_{0}:=\mathcal{F} u_{0}$, where the time Fourier transform $\mathcal{F}$ of a function $v$ defined on $\mathbb{R}$ is given by

$$
\hat{v}:=\mathcal{F} v(x, \xi):=\int_{\mathbb{R}} v(x, t) e^{-2 i \pi \xi t} d t .
$$


From (6), one gets

$$
\begin{aligned}
\widehat{u}_{0}= & \frac{A}{2 i \sin \left(\kappa_{\mathrm{f}} L\right)} \sin \left(\kappa_{\mathrm{f}}(L-x)\right)\left(\delta_{\nu_{m}}-\delta_{-\nu_{m}}\right) \\
& +\sum_{m \in \mathbb{N}} A \frac{\kappa_{\mathrm{f}}}{i L\left(\kappa_{\mathrm{f}}^{2}-\kappa_{m}^{2}\right)} \sin \left(\kappa_{m} x\right)\left(\delta_{\nu_{m}}-\delta_{-\nu_{m}}\right) \\
=: & \widehat{u}_{0, \nu_{\mathrm{f}}}+\sum_{m \in \mathbb{N}} \widehat{u}_{0, \nu_{m}},
\end{aligned}
$$

where $\delta_{a}$ is the Dirac distribution at a point $a$. Considering (8), we observe that the amplitude of the $m$-th mode $\widehat{u}_{0, \nu_{m}}$ scales like $\kappa_{\mathrm{f}} / L\left|\kappa_{\mathrm{f}}^{2}-\kappa_{m}^{2}\right|$, and thus exhibits a fast decay as the frequency $\nu_{m}$ moves away from $\nu_{\mathrm{f}}$ (see e.g. Figure 1 for an example of such a solution). When $\nu_{\mathrm{f}}$ is close to an eigenfrequency $\nu_{m}$, then the solution tends to behave like a single-mode with infinite amplitude, hence concentrating the energy of the system.

In the case of a moving boundary, we expect that the overall behavior of the solution owns similar properties: a first main contribution centered around the frequency $\nu_{\mathrm{f}}$ and a finite discrete sum of other significant contributions centered around the resonances $\nu_{m}$, most particularly for a small perturbation of the boundary oscillating at a frequency $\nu_{\ell}$ such that $0<\nu_{\ell} \ll \nu_{\mathrm{f}}$. However, in practical applications, the domain is often partially open and/or has wave absorbing objects at the boundary, which leads to non resonant finite amplitude solutions. This situation is investigated in Section 6 for the two- and three-dimensional cases. For practical remote sensing applications based on the Doppler effect, the useful information is mainly related to perturbations around $\nu_{\mathrm{f}}$.

\subsection{Reformulation of the initial problem as a fixed domain prob- lem}

Let us now analyze the case of a moving boundary. We first map $\Omega(t)$ to the fixed domain $\widetilde{\Omega}=] 0, L[$. This results in a new wave-like equation from the metric change. The physical solution $u$ is obtained by applying the reverse change of variable, i.e. from $\widetilde{\Omega}$ to $\Omega(t)$. More precisely, the change of space variable $\widetilde{x}:(x, t) \mapsto \widetilde{x}(x, t)$ is such that $\widetilde{x}(0, t)=0$, $\widetilde{x}(\ell(t), t)=L$, for all $t>0$, and is built as a smooth mapping with respect to $x$ and $t$. The spatial and time derivative operators then write

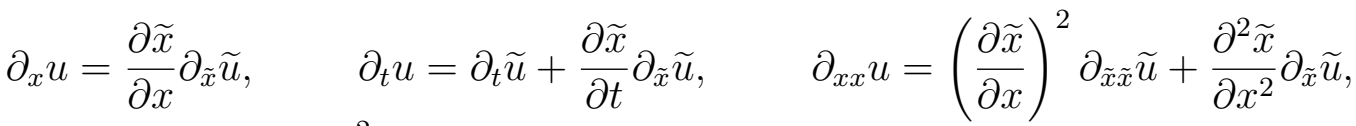

$$
\begin{aligned}
& \partial_{t t} u=\partial_{t t} \widetilde{u}+\left(\frac{\partial \widetilde{x}}{\partial t}\right)^{2} \partial_{\tilde{x} \tilde{x}} \widetilde{u}+2 \frac{\partial \widetilde{x}}{\partial t} \partial_{t \tilde{x}} \widetilde{u}+\frac{\partial^{2} \widetilde{x}}{\partial t^{2}} \partial_{\tilde{x}} \widetilde{u}
\end{aligned}
$$

Therefore, the initial scattering problem can be rewritten as follows: find $\widetilde{u}(\widetilde{x}, t)=u(x, t)$ that maps from $\widetilde{\Omega} \times \mathbb{R}_{*}^{+}$to $\mathbb{R}$ and that satisfies

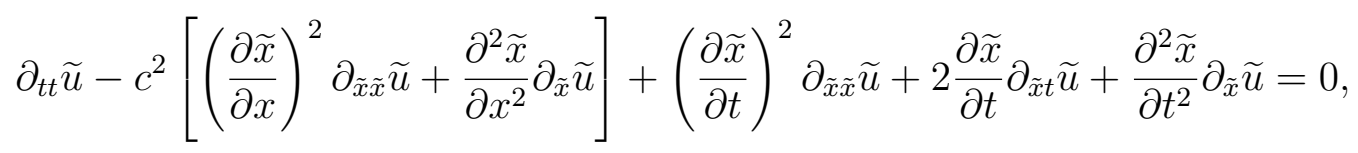


with $\widetilde{u}(0, t)=A \sin \left(\omega_{\mathrm{f}} t\right), \widetilde{u}(L, t)=0, \widetilde{u}(\widetilde{x}, 0)=0$ and

$$
\partial_{t} \widetilde{u}(\widetilde{x}, 0)=-\left.\frac{\partial \tilde{x}}{\partial t}\right|_{t=0} \partial_{\tilde{x}} \widetilde{u}(\widetilde{x}, 0)=0 .
$$

Let us remark that, similarly to [33], the spatial Jacobian $\frac{\partial \tilde{x}}{\partial x}$ of $\widetilde{x}$ appears in (9). In the following, we provide a frequency domain method to solve the modified wave equation (9).

\section{The specific case of a boundary with sine motion}

\subsection{Analysis for the case of a small amplitude boundary motion}

Let us consider the case where the motion of the right endpoint $\ell(t)$ of the domain $\Omega(t)$ is given by a small smooth time-dependent perturbation $L \epsilon f\left(\omega_{\ell} t\right)$ of $L$, where $f$ is a smooth bounded function with bounded derivatives $f^{(p)}$, oscillating with a frequency $\nu_{\ell}=\omega_{\ell} /(2 \pi)$ small compared to the emitter frequency $\nu_{\mathrm{f}}$, that is $0<\nu_{\ell} \ll \nu_{\mathrm{f}}$. Therefore, we have

$$
\ell(t)=L\left(1+\epsilon f\left(\omega_{\ell} t\right)\right)
$$

with $\epsilon \ll 1$. In the one-dimensional case, we can assume that this is given by a linear relation $x=\tilde{x} \ell(t) / L$. To be more explicit, we now focus on the special case where the boundary has a small time sinusoidal motion around $L$, defined by

$$
\ell(t)=L\left(1+\epsilon \sin \left(\omega_{\ell} t\right)\right)
$$

i.e. setting $f\left(\omega_{\ell} t\right):=\sin \left(\omega_{\ell} t\right)$. This leads to

$$
\begin{gathered}
\frac{\partial \widetilde{x}}{\partial x}=\frac{1}{1+\epsilon \sin \left(\omega_{\ell} t\right)}=\mathcal{O}(1), \quad \frac{\partial \widetilde{x}}{\partial t}=-\widetilde{x} \frac{\epsilon \omega_{\ell} \cos \left(\omega_{\ell} t\right)}{1+\epsilon \sin \left(\omega_{\ell} t\right)}=\mathcal{O}(\epsilon), \\
\frac{\partial^{2} \widetilde{x}}{\partial x^{2}}=0, \quad \frac{\partial^{2} \widetilde{x}}{\partial t^{2}}=\widetilde{x} \frac{\epsilon \omega_{\ell}^{2}\left(\sin \left(\omega_{\ell} t\right)\left(1+\epsilon \sin \left(\omega_{\ell} t\right)\right)-2 \epsilon \cos \left(\omega_{\ell} t\right)\right)}{\left(1+\epsilon \sin \left(\omega_{\ell} t\right)\right)^{2}}=\mathcal{O}(\epsilon) .
\end{gathered}
$$

Neglecting the $\mathcal{O}(\epsilon)$ terms in (9), we obtain the variable speed wave equation

$$
\partial_{t t} \widetilde{v}-c^{2}\left(\frac{\partial \widetilde{x}}{\partial x}\right)^{2} \partial_{\tilde{x} \tilde{x}} \widetilde{v}=0,
$$

which can be written as

$$
\frac{\ell^{2}}{(L c)^{2}} \partial_{t t} \widetilde{v}-\partial_{\tilde{x} \tilde{x}} \widetilde{v}=0
$$

in $\widetilde{\Omega} \times \mathbb{R}_{*}^{+}$, with $\widetilde{v}(0, t)=A \sin \left(\omega_{\mathrm{f}} t\right), \widetilde{v}(L, t)=0, \widetilde{v}(\widetilde{x}, 0)=0$ and $\partial_{t} \widetilde{v}(\widetilde{x}, 0)=0$.

Let us denote by $\square$ the d'Alembert operator

$$
\square:=\frac{1}{c^{2}} \partial_{t t}-\partial_{\tilde{x} \tilde{x}}
$$


and $u_{0}(\widetilde{x}, t)$ as the solution to (1) in the case of fixed boundaries. We consider the modified operator

$$
\square_{\epsilon}:=\frac{\ell(t)^{2}}{(L c)^{2}} \partial_{t t}-\partial_{\tilde{x} \tilde{x}}=\square+\epsilon \square_{1}
$$

where

$$
\square_{1}:=\frac{2 \sin \left(\omega_{\ell} t\right)+\epsilon \sin \left(\omega_{\ell} t\right)^{2}}{c^{2}} \partial_{t t} .
$$

For a solution $\square_{\epsilon} \widetilde{v}=1$, we define the perturbative part $\widetilde{v}_{1}$ by $\widetilde{v}=u_{0}+\widetilde{v}_{1}$, leading to

$$
\square_{\epsilon} \widetilde{v}_{1}=-\epsilon \square_{1} u_{0}=-\epsilon \square_{1}\left(u_{0, \nu_{\mathrm{f}}}+\sum_{m \in \mathbb{N}} u_{0, \nu_{m}}\right),
$$

according to (6).

Let us analyze the solution to the equations associated to each single source term involved in the series expansion of $\widetilde{v}_{0}$, i.e.

$$
\square_{\epsilon} \widetilde{v}_{1, \nu_{\mathrm{f}}}=-\epsilon \square_{1} u_{0, \nu_{\mathrm{f}}}
$$

and, for $m \in \mathbb{N}$,

$$
\square_{\epsilon} \widetilde{v}_{1, \nu_{m}}=-\epsilon \square_{1} u_{0, \nu_{m}} .
$$

The right hand sides are $\mathcal{C}^{\infty}\left(\widetilde{\Omega} \times \mathbb{R}_{*}^{+}\right)$functions and the corresponding boundary conditions are homogeneous Dirichlet conditions. Thus, since these equations are of hyperbolictype, we have the existence and uniqueness of the solutions and by superposition

$$
\widetilde{v}_{1}=\widetilde{v}_{1, \nu_{\mathrm{f}}}+\sum_{m \in \mathbb{N}} \widetilde{v}_{1, \nu_{m}}
$$

Finally, we prove that there exist some functions $\widetilde{v}_{\nu_{\mathrm{f}}}$ and $\widetilde{v}_{\nu_{m}}, m \in \mathbb{N}$, in $\mathcal{C} \infty\left(\widetilde{\Omega} \times \mathbb{R}_{*}^{+}\right)$such that the solution $\widetilde{v}$ of $(13)$ admits the following decomposition:

$$
\widetilde{v}=\widetilde{v}_{\nu_{\mathrm{f}}}+\sum_{m \in \mathbb{N}} \widetilde{v}_{\nu_{m}}
$$

with $\widetilde{v}_{\nu_{\mathrm{f}}}=u_{0, \nu_{\mathrm{f}}}+\widetilde{v}_{1, \nu_{\mathrm{f}}}$ and $\widetilde{v}_{\nu_{m}}=u_{0, \nu_{m}}+\widetilde{v}_{1, \nu_{m}}$, for $m \in \mathbb{N}$.

This form provides a generalization of (6) to the case of a moving boundary based on the approximation (13) of the full wave equation (9). Let us also remark that the previous decomposition can be extended to the case of smooth and bounded functions $\ell$ defined by a function $f$.

We now focus on the term $\widetilde{v}_{\nu_{\mathrm{f}}}$ appearing in the expansion (14). A similar approach can be adapted to the other terms $\widetilde{v}_{\nu_{m}}$, for $m \in \mathbb{N}$. Based on the method of separation of variables, we define $\widetilde{v}_{\nu_{\mathrm{f}}}(\widetilde{x}, t)=\widetilde{E}(\widetilde{x}) \widetilde{V}(t)$. The time-dependent component $\widetilde{V}(t)$ of $\widetilde{v}_{\nu_{\mathrm{f}}}(\widetilde{x}, t)$ is given as the solution of the variable coefficients ODE

$$
\partial_{t t} \widetilde{V}+\frac{\omega_{\mathrm{f}}^{2} L^{2}}{\ell(t)^{2}} \widetilde{V}=0,
$$


for which we are seeking a solution in $\mathcal{C}^{\infty}\left(\mathbb{R}_{*}^{+}\right)$. Since

$$
\frac{\omega_{\mathrm{f}}^{2} L^{2}}{\ell(t)^{2}}=\frac{\omega_{\mathrm{f}}^{2}}{\left(1+\epsilon \sin \left(\omega_{\ell} t\right)\right)^{2}},
$$

and $\left.\epsilon \sin \left(\omega_{\ell} t\right) \in\right]-1,1[$, we have the power series expansion

$$
\begin{aligned}
\frac{\omega_{\mathrm{f}}^{2} L^{2}}{\ell(t)^{2}} & =\omega_{\mathrm{f}}^{2} \sum_{k \in \mathbb{N}}(-1)^{k}(k+1)\left(\epsilon \sin \left(\omega_{\ell} t\right)\right)^{k} \\
& =\omega_{\mathrm{f}}^{2}\left(1-2 \epsilon \sin \left(\omega_{\ell} t\right)+\mathcal{O}\left(\epsilon^{2}\right)\right) .
\end{aligned}
$$

For $\epsilon \ll 1$, we consider the approximate solution $\widetilde{W}$ to the equation $(15)$ based on the first-order truncation of (16), i.e.

$$
\partial_{t t} \widetilde{W}+\omega_{\mathrm{f}}^{2}\left(1-2 \epsilon \sin \left(\omega_{\ell} t\right)\right) \widetilde{W}=0 .
$$

Let us define the change of variable $\tau:=\pi \nu_{\ell} t+\frac{\pi}{4}$ and the function $\bar{W}(\tau):=\widetilde{W}\left(\pi \nu_{\ell} t+\frac{\pi}{4}\right)$. Then the second-order derivative writes

$$
\partial_{t t} \widetilde{W}=\left(\pi \nu_{\ell}\right)^{2} \partial_{\tau \tau} \bar{W}
$$

From (17), we deduce the Mathieu's equation

$$
\partial_{\tau \tau} \bar{W}+(\alpha-2 q \cos (2 \tau)) \bar{W}=0
$$

with $\alpha=\left(\frac{2 \nu_{\mathrm{f}}}{\nu_{\ell}}\right)^{2}$ and $q=-\alpha \epsilon$. The solutions of 18 are given as linear combinations of Mathieu functions of the first-kind [1]. According to Floquet's theory [37], they can be written as $\phi(\tau)=e^{i \mu \tau} p(\tau)$, with $p$ a $\pi$-periodic function and $\mu$ the associated Floquet's exponent. Let us remark that the function $\phi(-\tau)$ is also solution to the equation (18). Whenever $\phi(\tau)$ and $\phi(-\tau)$ are linearly independent, the general solution writes [1] as

$$
\begin{aligned}
\bar{W}(\tau) & =e^{i \mu \tau} \sum_{j \in \mathbb{Z}} \bar{a}_{j}^{+} e^{2 i j \tau}+e^{-i \mu \tau} \sum_{j \in \mathbb{Z}} \bar{a}_{j}^{-} e^{-2 i j \tau} \\
& =\sum_{j \in \mathbb{Z}} \bar{a}_{j}^{+} e^{2 i\left(\frac{\mu}{2}+j\right) \tau}+\bar{a}_{j}^{-} e^{-2 i\left(\frac{\mu}{2}+j\right) \tau}
\end{aligned}
$$

with $\bar{a}_{j}^{ \pm}$being the complex valued Fourier coefficients of $p$. Since $\bar{W}(\tau)$ is a periodic $\mathcal{C}^{\infty}\left(\mathbb{R}_{*}^{+}\right)$ function, $\left|\bar{a}_{j}^{ \pm}\right|$has a fast decay when $|j| \rightarrow+\infty$.

As $q=\mathcal{O}(\epsilon)$, for sufficiently small perturbations $\epsilon$, we may expand $\alpha$ in terms of $\mu$ and $q$ (cf. [1], ch. 20, p. 730)

$$
\alpha=\mu^{2}+\frac{q^{2}}{2\left(\mu^{2}-1\right)}+\mathcal{O}\left(\frac{q^{4}}{\mu^{6}}\right)=\mu^{2}+\mathcal{O}\left(\epsilon^{2}\right)
$$


Hence, at first-order in $\epsilon$, one gets $\mu \approx \sqrt{\alpha}=\frac{2 \nu_{f}}{\nu_{\ell}}$, and, for small values of $\epsilon$, we have the approximation

$$
\bar{W}(\tau) \approx \sum_{j \in \mathbb{Z}} \bar{a}_{j}^{+} e^{2 i\left(\frac{\nu_{\mathrm{f}}}{\nu_{\ell}}+j\right) \tau}+\bar{a}_{j}^{-} e^{-2 i\left(\frac{\nu_{\mathrm{f}}}{\nu_{\ell}}+j\right) \tau},
$$

which yields, for $\tau=\pi \nu_{\ell} t+\frac{\pi}{4}$,

$$
\widetilde{W}(t) \approx \sum_{j \in \mathbb{Z}} i^{j} \bar{a}_{j}^{+} e^{i \frac{\pi \nu_{\mathrm{f}}}{2 \nu_{\ell}}} e^{2 i \pi\left(\nu_{\mathrm{f}}+j \nu_{\ell}\right) t}+(-i)^{j} \bar{a}_{j}^{-} e^{-i \frac{\pi \nu_{\mathrm{f}}}{2 \nu_{\ell}}} e^{-2 i \pi\left(\nu_{\mathrm{f}}+j \nu_{\ell}\right) t}
$$

Combining $( \pm i)^{j} \bar{a}_{j}^{ \pm} e^{ \pm i \frac{\pi \nu_{\mathrm{f}}}{2 \nu_{\ell}}}$ with the space dependent component $\widetilde{E}(\widetilde{x})$ of $\widetilde{v}$, we define the coefficients $\left\{\widetilde{a}_{j}^{ \pm}(\widetilde{x})\right\}_{j \in \mathbb{Z}}$ such that, for each integer $j$, we have

$$
\widetilde{a}_{j}^{ \pm}(\widetilde{x})=( \pm i)^{j} \bar{a}_{j}^{ \pm} e^{ \pm i \frac{\pi \nu_{\mathrm{f}}}{2 \nu_{\ell}}} \widetilde{E}(\widetilde{x})
$$

We formally obtain the following ansatz for small $\epsilon$

$$
\widetilde{v}_{\nu_{\mathrm{f}}}(\widetilde{x}, t) \approx \sum_{j \in \mathbb{Z}} \widetilde{a}_{j}^{+}(\widetilde{x}) e^{i\left(\omega_{\mathrm{f}}+j \omega_{\ell}\right) t}+\widetilde{a}_{j}^{-}(\widetilde{x}) e^{-i\left(\omega_{\mathrm{f}}+j \omega_{\ell}\right) t} .
$$

Let us define, for fixed integers $J_{1} \leq 0$ and $J_{2} \geq 0$ the finite sets of integers $\mathcal{I}=\mathcal{I}^{-} \cup \mathcal{I}^{+}$ and $\mathcal{J}=\mathcal{J}^{-} \cup \mathcal{J}^{+}$, where

$$
\begin{array}{ll}
\mathcal{I}^{-}:=\left\{j \in \mathbb{Z},-J_{2} \leq j \leq-J_{1}\right\}, & \mathcal{I}^{+}:=\left\{j \in \mathbb{Z}, J_{1} \leq j \leq J_{2}\right\} \\
\mathcal{J}^{-}:=\left\{-\nu_{\mathrm{f}}+\nu_{\ell} j\right\}_{j \in \mathcal{I}^{-}}, & \mathcal{J}^{+}:=\left\{\nu_{\mathrm{f}}+\nu_{\ell} j\right\}_{j \in \mathcal{I}^{+}}
\end{array}
$$

We denote by $\sharp \mathcal{I}$ the cardinal of the set $\mathcal{I}$. Since $\left|\widetilde{a}_{j}^{ \pm}(\widetilde{x})\right|$ is fastly decaying for $|j| \rightarrow \infty,(21)$ justifies that the method that we develop (most particularly for small $\epsilon$ ) in the following is based on the approximation $\widetilde{w}^{\mathcal{I}}$ of $\widetilde{v}_{\nu_{\mathrm{f}}}$ by the finite sum

$$
\widetilde{w}^{\mathcal{I}}(\widetilde{x}, t):=\sum_{j \in \mathcal{I}^{+}} \widetilde{a}_{j}^{+}(\widetilde{x}) e^{i\left(\omega_{\mathrm{f}}+j \omega_{\ell}\right) t}+\widetilde{a}_{j}^{-}(\widetilde{x}) e^{-i\left(\omega_{\mathrm{f}}+j \omega_{\ell}\right) t},
$$

where $\sharp \mathcal{I}=2 \sharp \mathcal{I}^{+}$coefficients $\widetilde{a}_{j}^{ \pm}(\widetilde{x})$ have to be computed thanks to a coupled system of multi-harmonic Helmholtz-type equations in the Fourier domain. The two integers $J_{1}$ and $J_{2}$ must be carefully chosen to include the significant contributions to the solution.

By construction of the Fourier series expansion, we have $\widetilde{a}_{j}^{+}=\overline{\widetilde{a}_{j}^{-}}$. Thus, one gets

$$
\begin{aligned}
\widetilde{w}^{\mathcal{I}}(\widetilde{x}, t) & =\sum_{j \in \mathcal{I}^{+}} \widetilde{a}_{j}^{+}(\widetilde{x}) e^{i\left(\omega_{\mathrm{f}}+j \omega_{\ell}\right) t}+\sum_{j \in \mathcal{I}^{+}} \overline{\widetilde{a}_{j}^{+}}(\widetilde{x}) e^{-i\left(\omega_{\mathrm{f}}+j \omega_{\ell}\right) t} \\
& =: \widetilde{w}^{\mathcal{I}^{+}}(\widetilde{x}, t)+\widetilde{w}^{\mathcal{I}^{-}}(\widetilde{x}, t),
\end{aligned}
$$

where the Fourier coefficients of $\widetilde{w}^{\mathcal{I}^{-}}$are the complex conjugates of the Fourier coefficients of $\widetilde{w}^{\mathcal{I}^{+}}$.

Let us remark that the expression (14) of $\widetilde{v}$ also involves the functions $\widetilde{v}_{\nu_{m}}$. However, their contribution to $\widetilde{v}$ is less significant than $\widetilde{v}_{\nu_{\mathrm{f}}}$. In the case where a part of the domain is open or includes some absorption materials (see Section 6), they are even negligible. Finally, if $\epsilon=0$, all the above computations are exact and $J_{1}=J_{2}=0$. 


\subsection{Derivation of a multi-harmonic approximate system}

Let us assume that the Leibniz integral rule holds for $\widetilde{v}$. Then, assuming that the time Fourier transform $\widehat{v}$ of $\widetilde{v}$ is defined according to (7) (with the extension $\widetilde{v}=u_{0}$ for $t<0$ ), one gets: $\mathcal{F} \partial_{\tilde{x} \tilde{x}} \widetilde{v}(\widetilde{x}, t)=\partial_{\tilde{x} \tilde{x}} \widehat{v}(\widetilde{x}, \xi)$, setting $\widehat{v}(\widetilde{x}, \xi):=\mathcal{F} \widetilde{v}(\widetilde{x}, t)$.

Applying $\mathcal{F}$ to 13 - 11 gives

$$
\begin{aligned}
\mathcal{F}\left(\partial_{\tilde{x} \tilde{x}} \widetilde{v}\right. & \left.-\frac{1}{c^{2}}\left[1+\frac{\epsilon^{2}}{2}+2 \epsilon \sin \left(\omega_{\ell} t\right)-\frac{\epsilon^{2}}{2} \cos \left(2 \omega_{\ell} t\right)\right] \partial_{t t} \widetilde{v}\right) \\
= & \partial_{\tilde{x} \tilde{x}} \widehat{v}+\left[\left(1+\frac{\epsilon^{2}}{2}\right) \delta_{0}+i \epsilon\left(\delta_{-\nu_{\ell}}-\delta_{\nu_{\ell}}\right)-\frac{\epsilon^{2}}{4}\left(\delta_{2 \nu_{\ell}}+\delta_{-2 \nu_{\ell}}\right)\right] *\left[\left(\frac{2 \pi \xi}{c}\right)^{2} \widehat{v}\right]=0,
\end{aligned}
$$

where $*$ is the convolution product. Denoting by $\tau_{a}$ the translation operator such that $\tau_{a} f(t)=f(t-a)$, we obtain the following problem: find $\widehat{v}: \widetilde{\Omega} \times \mathbb{R} \rightarrow \mathbb{R}$ satisfying

$$
\partial_{\tilde{x} \tilde{x}} \widehat{v}+\left[\left(1+\frac{\epsilon^{2}}{2}\right)+i \epsilon\left(\tau_{-\nu_{\ell}}-\tau_{\nu_{\ell}}\right)-\frac{\epsilon^{2}}{4}\left(\tau_{2 \nu_{\ell}}+\tau_{-2 \nu_{\ell}}\right)\right]\left[\left(\frac{2 \pi \xi}{c}\right)^{2} \widehat{v}\right]=0 .
$$

In addition, for all $\xi \in \mathbb{R}$, we have $\widehat{v}(L, \xi)=0, \widehat{v}(0, \xi)=0$ if $|\xi| \neq \nu_{\mathrm{f}}, \widehat{v}\left(0, \nu_{\mathrm{f}}\right)=\frac{A}{2 i}$ and $\widehat{v}\left(0,-\nu_{\mathrm{f}}\right)=-\frac{A}{2 i}$.

Let us now compute an approximation of the contribution $\widehat{v}_{\nu_{\mathrm{f}}}$ of the solution $\widehat{v}$ to 25 around $\nu_{\mathrm{f}}$. From the ansatz (23), it is reasonable to look for an approximate solution $\widehat{w}^{\mathcal{I}}(\widetilde{x}, \xi)$ of $\widehat{v}_{\nu_{\mathrm{f}}}(\widetilde{x}, \xi)$ as

$$
\widehat{w}^{\mathcal{I}}=\widehat{w}^{\mathcal{I}^{+}}+\widehat{w}^{\mathcal{I}^{-}}:=\sum_{j \in \mathcal{I}^{+}} \widetilde{a}_{j}^{+} \delta_{\nu_{\mathrm{f}}+j \nu_{\ell}}+\sum_{j \in \mathcal{I}^{+}} \overline{\widetilde{a}_{j}^{+}} \delta_{-\left(\nu_{\mathrm{f}}+j \nu_{\ell}\right)}
$$

which is a linear combination of Dirac distributions, centered around $\pm \nu_{\mathrm{f}}$, spaced by the modulating frequency $\nu_{\ell}$, and with (unknown) spatial amplitudes $\left\{\widetilde{a}_{j}^{ \pm}\right\}_{\mathcal{I}^{+}}$. The fast decay of the coefficients $\left|\widetilde{a}_{j}^{+}\right|$for $|j| \rightarrow+\infty$ guarantees that the finite supports $\mathcal{J}^{ \pm}$of the tempered distributions $\widehat{w}^{\mathcal{I}^{ \pm}}$are disjoint. Hence, we can restrict our analysis to the contribution $\widehat{w}^{\mathcal{I}^{+}}$. Plugging the expansion of $\widehat{w}^{\mathcal{I}^{+}}(\widetilde{x}, \xi)$ defined in 26 into 25 , and identifying the coefficients in front of the Dirac distributions leads to

$$
\partial_{\tilde{x} \tilde{x}} \widetilde{a}_{j}^{+}+\kappa_{j}^{2}\left(1+\frac{\epsilon^{2}}{2}\right) \widetilde{a}_{j}^{+}+i \epsilon\left(\kappa_{j+1}^{2} \widetilde{a}_{j+1}^{+}-\kappa_{j-1}^{2} \widetilde{a}_{j-1}^{+}\right)-\frac{\epsilon^{2}}{4}\left(\kappa_{j-2}^{2} \widetilde{a}_{j-2}^{+}+\kappa_{j+2}^{2} \widetilde{a}_{j+2}^{+}\right)=0,
$$

for $j \in \mathcal{I}^{+}$, with $\kappa_{j}:=\left(\omega_{\mathrm{f}}+j \omega_{\ell}\right) / c$ and for the boundary conditions $\widetilde{a}_{0}^{+}(0)=\frac{A}{2 i}, \widetilde{a}_{j}^{+}(0)=0$ for $j \neq 0, \widetilde{a}_{j}^{+}(L)=0$, and $\widetilde{a}_{j}^{+}(\widetilde{x})=0$ for $\widetilde{x} \in \Omega$ and $j \in \mathbb{Z} \backslash \mathcal{I}^{+}$.

Equation (27) describes the coupling between the frequency components induced by the boundary oscillations. As expected, taking $\epsilon=0$ gives a system of uncoupled Helmholtz equations, where the coefficients vanish for $j \neq 0$, and

$$
\widetilde{a}_{0}^{+}(\tilde{x})=\frac{A}{2 i} \frac{\sin \left(\kappa_{\mathrm{f}}(L-x)\right)}{\sin \left(\kappa_{\mathrm{f}} L\right)} .
$$


In Section 4, we numerically solve (27) by means of the finite element method. To this end, we derive the following coupled weak formulation: find $a_{j}^{*} \in \mathcal{H}_{\mathrm{D}_{j}}^{1}(\widetilde{\Omega}):=\left\{a_{j}^{*} \in\right.$ $\mathcal{H}^{1}(\widetilde{\Omega}) \mid a_{0}^{*}(0)=\frac{A}{2 i}, a_{j}^{*}(0)=0$ for $\left.j \neq 0, a_{j}^{*}(L)=0\right\}$ such that

$$
\begin{array}{r}
-\int_{\widetilde{\Omega}} \partial_{\tilde{x}} a_{j}^{*} \partial_{\tilde{x}} \phi d \widetilde{x}+\int_{\widetilde{\Omega}}\left[\kappa_{j}^{2}\left(1+\epsilon^{2}\right) a_{j}^{*}+i \epsilon\left(\kappa_{j+1}^{2} a_{j+1}^{*}-\kappa_{j-1}^{2} a_{j-1}^{*}\right)\right. \\
\left.-\epsilon^{2}\left(\kappa_{j-2}^{2} a_{j-2}^{*}+\kappa_{j+2}^{2} a_{j+2}^{*}\right)\right] \phi d \widetilde{x}=0
\end{array}
$$

holds for all test functions $\phi \in \mathcal{H}_{0}^{1}(\widetilde{\Omega})$ and for $j \in \mathcal{I}^{+}$, with $a_{j}^{*}(\widetilde{x})=0$ for $j \in \mathbb{Z} \backslash \mathcal{I}^{+}$. We then consider a regular covering $\widetilde{\Omega}_{h}$ of $\widetilde{\Omega}$ using $n_{\widetilde{\Omega}}$ finite elements of size $h$. All the notations are extended with an $h$ subscript for the discrete version of the domains as well as unknowns. We choose a linear element approximation and denote by

$$
\widehat{w}_{h}^{\mathcal{I}^{+}}=\sum_{j \in \mathcal{I}^{+}} a_{h, j}^{*} \delta_{\nu_{\mathrm{f}}+j \nu_{\ell}}
$$

the solution of the discretization of the variational coupled system $(28)$, with $a_{h, j}^{*}$ belonging to the $\left(n_{\widetilde{\Omega}}-1\right)$-dimensional finite element subspace $\mathcal{V}_{\mathrm{D}_{j}}$ of $\mathcal{H}_{\mathrm{D}_{j}}^{1}(\widetilde{\Omega})$.

\subsection{The case of a larger amplitude boundary motion}

The previous method has been specifically designed for the case of small amplitude motions, according to the approximate equation (27). In this subsection, we focus on a more general case as we transfer the exact equation (9) directly to the frequency domain. The range of validity of this approach is explored in a numerical study in Section 4.

For $\epsilon \in] 0, L[$ and a linear transformation in $x,(9)$ can be written as follows

$$
\ell^{2} \partial_{t t} \widetilde{u}+\left(\widetilde{x}^{2} \ell^{\prime 2}-c^{2} L^{2}\right) \partial_{\tilde{x} \tilde{x}} \widetilde{u}-2 \widetilde{x} \ell \ell^{\prime} \partial_{\tilde{x} t} \widetilde{u}+\widetilde{x}\left(2 \ell^{\prime 2}-\ell \ell^{\prime \prime}\right) \partial_{\tilde{x}} \widetilde{u}=0,
$$

with $\widetilde{u}(0, t)=A \sin \left(\omega_{\mathrm{f}} t\right), \widetilde{u}(L, t)=0$ for $t>0$, and $\widetilde{u}(\widetilde{x}, 0)=0, \partial_{t} \widetilde{u}(\widetilde{x}, 0)=0$, for $\widetilde{x} \in \widetilde{\Omega}$. The methodology presented in the previous subsection is now applied to $(29)$. Let $\widehat{u}(\widetilde{x}, \xi):=\mathcal{F}(\widetilde{u})(\widetilde{x}, \xi)$ be the time Fourier transform of a solution $u$ of $(29)$. We assume that $\widetilde{u}_{\nu_{\mathrm{f}}}$ admits a decomposition of the form (14) (see also Figure 1). Let us now focus on the component $\widehat{u}_{\nu_{\mathrm{f}}}$ of $\widehat{u}$ and search for an approximation $\widehat{w}_{\text {gen }}^{\mathcal{I}^{+}}$of $\widehat{u}_{\nu_{\mathrm{f}}}$ for $\xi \in \mathcal{J}^{+}$, in the form of the ansatz 26 with coefficients $\widetilde{a}_{j, \text { gen }}^{+}$. 
Defining $\omega_{j}=1+j \omega$, this leads to the following coupled system of equations:

$$
\begin{aligned}
& \omega_{j}^{2}\left(1+\frac{\epsilon^{2}}{2}\right) \widetilde{a}_{j, \text { gen }}^{+}-\frac{3 \widetilde{x} \epsilon^{2} \omega^{2}}{2} \partial_{\tilde{x}} \widetilde{a}_{j, \text { gen }}^{+}-\left(\frac{\widetilde{x}^{2} \epsilon^{2} \omega^{2}}{2}-\frac{1}{\kappa_{\mathrm{f}}^{2}}\right) \partial_{\tilde{x} \tilde{x}} \widetilde{a}_{j, \text { gen }}^{+} \\
& +i \epsilon\left[\omega_{j+1}^{2} \widetilde{a}_{j+1, \text { gen }}^{+}-\omega_{j-1}^{2} \widetilde{a}_{j-1, \mathrm{gen}}^{+}+\widetilde{x} \omega\left(\omega_{j+\frac{1}{2}} \partial_{\tilde{x}} \widetilde{a}_{j+1, \text { gen }}^{+}+\omega_{j-\frac{1}{2}} \partial_{\tilde{x}} \widetilde{a}_{j-1, \mathrm{gen}}^{+}\right)\right] \\
& -\frac{\epsilon^{2}}{4}\left[\omega_{j-2}^{2} \widetilde{a}_{j-2, \mathrm{gen}}^{+}+\omega_{j+2}^{2} \widetilde{a}_{j+2, \mathrm{gen}}^{+}-2 \widetilde{x} \omega\left(\omega_{j-\frac{5}{2}} \partial_{\tilde{x}} \widetilde{a}_{j-2, \mathrm{gen}}^{+}-\omega_{j+\frac{5}{2}} \partial_{\tilde{x}} \widetilde{a}_{j+2, \mathrm{gen}}^{+}\right)\right. \\
& \left.+\widetilde{x}^{2} \omega^{2}\left(\partial_{\tilde{x} \tilde{x}} \widetilde{a}_{j-2, \mathrm{gen}}^{+}+\partial_{\tilde{x} \tilde{x}} \widetilde{a}_{j+2, \mathrm{gen}}^{+}\right)\right]=0
\end{aligned}
$$

for $j \in \mathcal{I}^{+}$, with $\omega=\omega_{\ell} / \omega_{\mathrm{f}}$, and with the same boundary conditions as for system (27). Let us remark that, based on (30), different asymptotics could be analyzed, based on $\epsilon, \omega$, or even $\epsilon \omega$.

As previously, in order to solve (30) numerically by means of the finite element method, we consider the associated weak formulation, which writes: find $a_{j, \text { gen }}^{*} \in \mathcal{H}_{D_{j}}^{1}(\widetilde{\Omega})$ such that

$$
\begin{gathered}
\int_{\widetilde{\Omega}}\left[\left(\frac{\epsilon^{2} \omega^{2} \widetilde{x}^{2}}{2}-\frac{1}{\kappa_{\mathrm{f}}^{2}}\right) \partial_{\tilde{x}} a_{j, \text { gen }}^{*} \partial_{\tilde{x}} \phi+\frac{\epsilon^{2} \widetilde{x} \omega^{2}}{4}\left(\partial_{\tilde{x}} a_{j+2, \text { gen }}^{*}+\partial_{\tilde{x}} a_{j-2, \text { gen }}^{*}\right) \partial_{\tilde{x}} \phi\right] d \widetilde{x} \\
-\int_{\widetilde{\Omega}} \frac{\epsilon \widetilde{x} \omega}{2}\left[\epsilon \omega \partial_{\tilde{x}} a_{j, \text { gen }}^{*} \phi-2 i\left(\omega_{j+\frac{1}{2}} \partial_{\tilde{x}} a_{j+1, \text { gen }}^{+}+\omega_{j-\frac{1}{2}} \partial_{\tilde{x}} a_{j-1, \text { gen }}^{+}\right) \phi\right. \\
\left.+\epsilon\left(\omega_{j+\frac{3}{2}} \partial_{\tilde{x}} a_{j+2, \text { gen }}^{*}-\omega_{j-\frac{3}{2}} \partial_{\tilde{x}} a_{j-2, \text { gen }}^{*}\right) \phi\right] d \widetilde{x} \\
+\int_{\widetilde{\Omega}}\left[\omega_{j}^{2}\left(1+\frac{\epsilon^{2}}{2}\right) a_{j, \text { gen }}^{*} \phi+i \epsilon\left(\omega_{j+1}^{2} a_{j+1, \text { gen }}^{*}-\omega_{j-1}^{2} a_{j-1, \text { gen }}^{*}\right) \phi\right. \\
\left.\quad-\frac{\epsilon^{2}}{4}\left(\omega_{j+2}^{2} a_{j+2, \text { gen }}^{*}+\omega_{j-2}^{2} a_{j-2, \text { gen }}^{*}\right) \phi\right] d \widetilde{x}=0
\end{gathered}
$$

holds for all test functions $\phi \in \mathcal{H}_{0}^{1}(\widetilde{\Omega})$ and $j \in \mathcal{I}^{+}$. We then use the same covering $\widetilde{\Omega}_{h}$ of $\widetilde{\Omega}$ and denote by $\widehat{w}_{\text {gen }, h}^{\mathcal{I}^{+}}$the solution of the discretization of the variational coupled system (31).

Before proceeding to extensive numerical tests that will illustrate our approach, we report in Figure 1 the amplitude spectrum of the numerical solution $\widehat{u}_{h}$ of $(29)$ at a fixed space point $\widetilde{x}=0.5(L=1)$, without moving boundary $(\epsilon=0$, in red, where $(29)$ is equivalent to (1) and $\left.\widehat{u} \equiv \widehat{u}_{0}\right)$ and with moving boundary $(\epsilon=0.1$, in blue). Concretely, the solution $\widehat{u}_{h}$ is computed by solving (29) numerically (with a second-order finite difference scheme in time and linear finite elements in space), leading to $\widetilde{u}_{h}$, and then applying the FFT. The physical parameters are $A=1, c=300, \nu_{\mathrm{f}}=360$ and $\nu_{\ell}=2$. The frequencies of the eigenmodes $\widehat{u}_{0, \nu_{m}}$ are $\nu_{m}=m c / 2$ and correspond to the peaks of the red curve. For $\epsilon=0$, we observe that i) there is a main contribution for $\nu_{\mathrm{f}}=360$ associated to $\widehat{u}_{0, \nu_{\mathrm{f}}}$ and ii) we have some uniformly distributed contributions $\widehat{u}_{0, \nu_{m}}$. This is in line with what is stated 


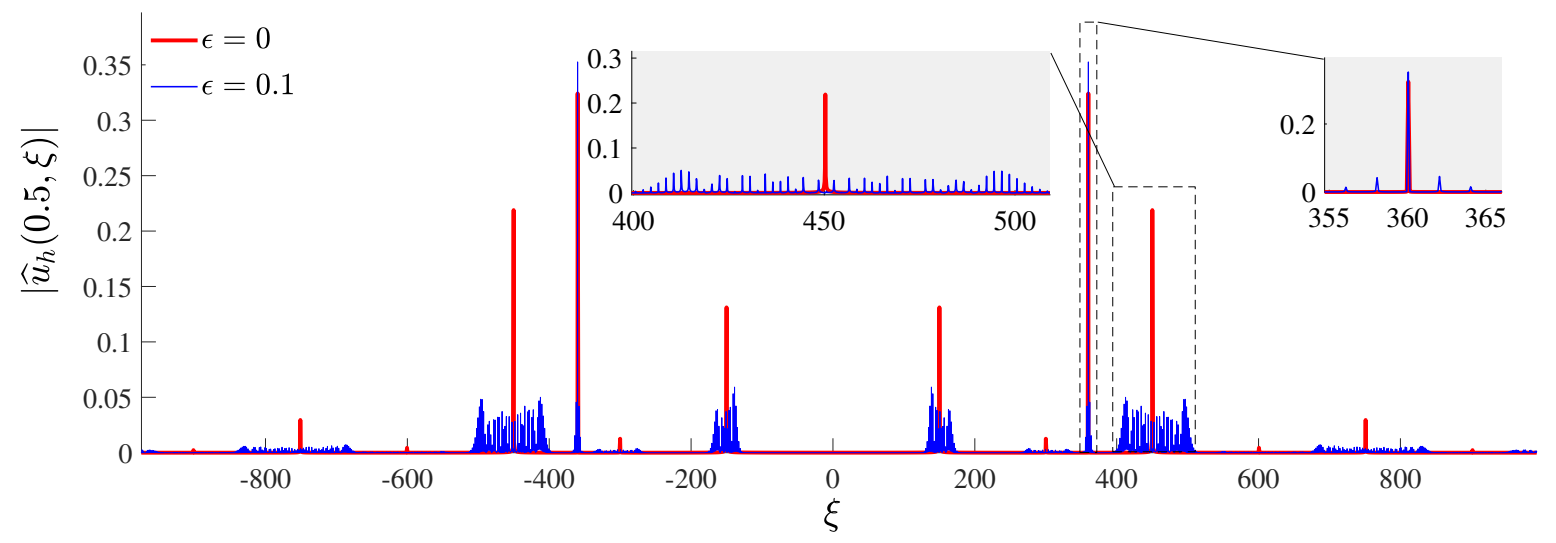

Fig. 1. Frequency spectrum $\left|\widehat{u}_{h}(0.5, \xi)\right|$ without $(\epsilon=0$, in red) and with boundary motion $(\epsilon=0.1$, in blue). This illustrates both the assumption on the decomposition (14) of $\widetilde{u}$ and the ansatz 26 .

in Proposition 1. In the case of the oscillating boundary $(\epsilon=0.1)$, we see again some contributions related to $\nu_{\mathrm{f}}$ and $\nu_{m}$ but with some additional new excited modes, localized around these frequencies and spaced by $\nu_{\ell}$.

Let us recall that the contributions $u_{\nu_{m}}, m \in \mathbb{N}$, are not significant in practice since usually the domain involves some open boundary parts and some dissipation effects. Here, since we have a Dirichlet boundary condition, we assume that $\nu_{\mathrm{f}}$ is not close to an eigenfrequency $\nu_{m}$. Therefore, the restriction of $\widehat{u}_{h}$ to $\mathcal{J}$ denoted by $\widehat{u}_{h}^{\mathcal{J}}$ is expected to provide a relatively accurate approximation of $\widehat{u}_{\nu_{\mathrm{f}}}$. To illustrate this claim, we report on Figure $2 \mathrm{a}$ the comparison between $\widetilde{u}_{h}$ and $\widetilde{u}_{h}^{\mathcal{J}}$ (defined as the inverse Fourier transform of $\widehat{u}_{h}^{\mathcal{J}}$ ) at $\widetilde{x}=0.5$, computed by means of iFFT. We observe that the phases of the two functions are in agreement and the envelope of the signal is well reproduced, with same frequency $\nu_{\ell}=2$. The difference between the signals is a consequence of the boundary movement.

The second approximation consists in validating the computation of $\widehat{u}^{\mathcal{J}}$ by the approximation $\widetilde{w}_{\text {gen }}^{\mathcal{I}}$. To this end, we report in Fig. $2 \mathrm{~b}$ the absolute error $\left.\mid \widetilde{u}_{h}^{\mathcal{J}}(0.5, t)-\widetilde{w}_{\text {gen }, h}^{\mathcal{I}}(0.5, t)\right]$ for the same configuration. We observe a good agreement between the solutions, which confirms that the expected ansatz $\sqrt{26}$ ) is valid for $\widetilde{w}_{\text {gen }}^{\mathcal{I}}$.

The values of $J_{1}$ and $J_{2}$ that define the interval $\mathcal{I}$ can be justified a posteriori by energy or numerical criteria but their a priori determination remains an open question. In the case of a general boundary motion, the choice of $J_{1}$ and $J_{2}$ is also related to the frequency of the input signal and the perturbation amplitude $\epsilon$. Indeed, we observe that the coefficients $\left|\widetilde{a}_{j, \text { gen }}^{ \pm}\right|$decay slower for increasing $|j|$ as $\epsilon$ gets larger.

Consequently, the values of $-J_{1}$ and $J_{2}$ used to truncate (30) have to be increased, as expected. We illustrate this property in Figure 3 where we report the amplitude of $\widehat{u}_{h}(0.5, \xi)$ computed by the brute force method, for $\nu_{\mathrm{f}}=3250$ and $c=1280$. The values of $\epsilon$ vary from 0.01 to 0.1 . 


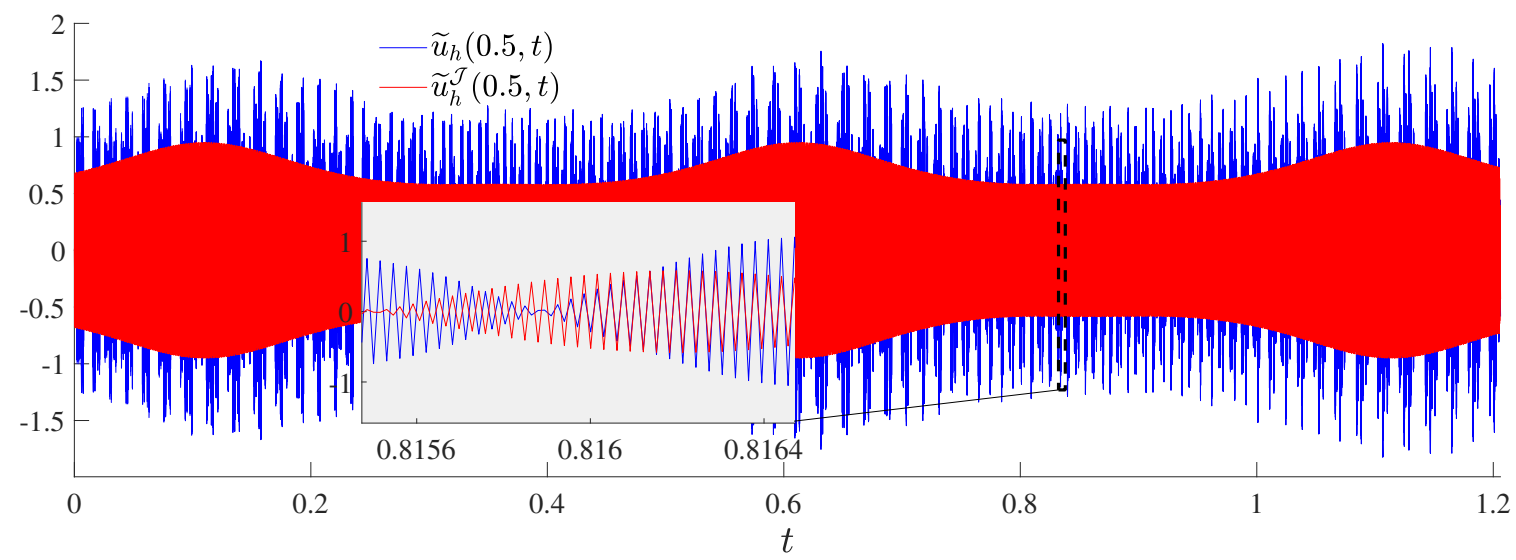

(a) $\widetilde{u}_{h}(0.5, t)$ vs. $t$ (blue) and $\widetilde{u}_{h}^{\mathcal{J}}(0.5, t)$ vs. $t$ (red).

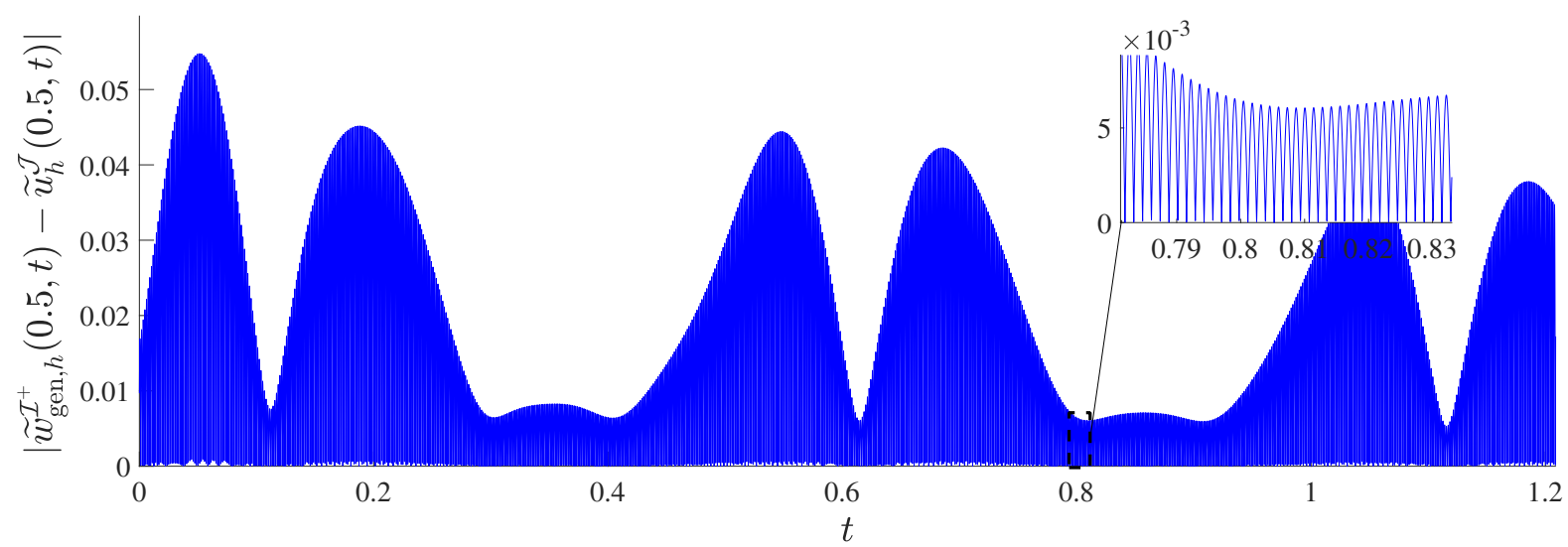

(b) $\left.\mid \widetilde{u}_{h}^{\mathcal{J}}(0.5, t)-\widetilde{w}_{\text {gen }, h}^{\mathcal{I}}(0.5, t)\right]$ vs. $t$.

Fig. 2. On the top figure, time signals $\widetilde{u}_{h}(0.5, t)$ in blue and $\widetilde{u}_{h}^{\mathcal{J}}(0.5, t)$ in red. On the bottom figure, their absolute difference vs. $t$. The physical parameters are $\nu_{\mathrm{f}}=360, \nu_{\ell}=2, c=300$ and $\epsilon=0.1$. 


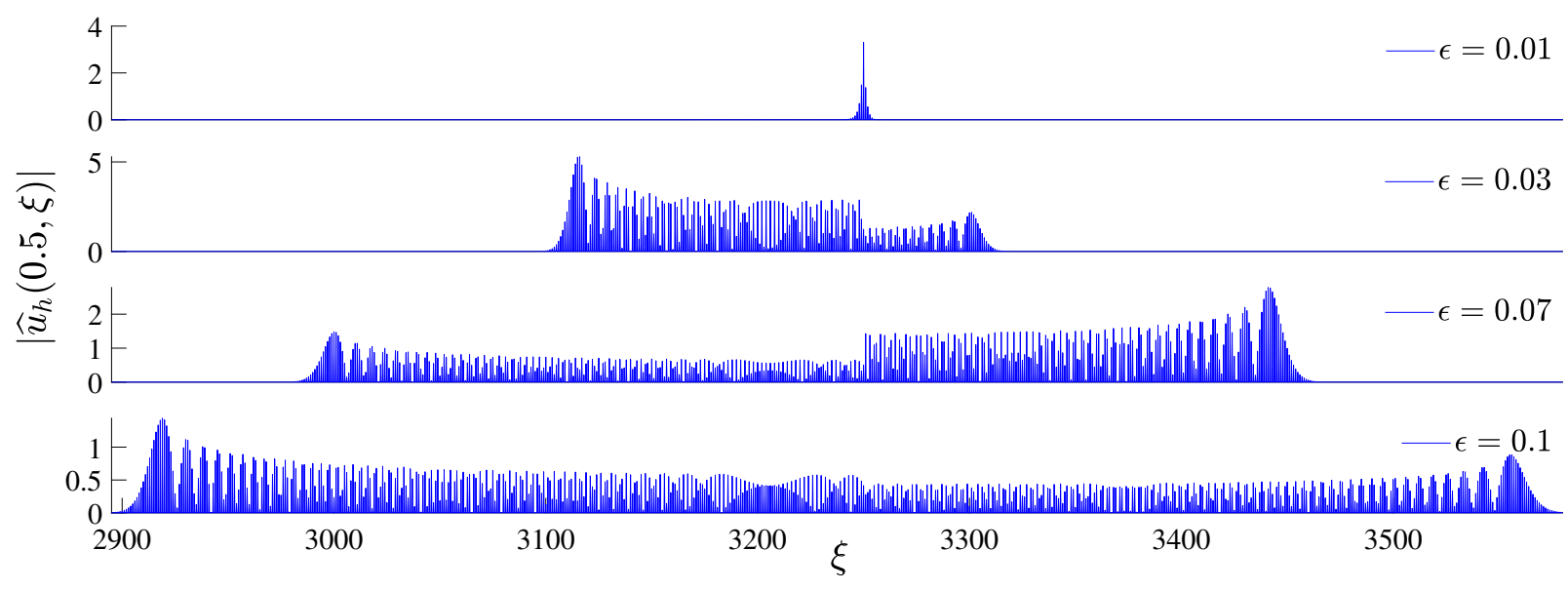

Fig. 3. Increasing the amplitude $\epsilon$ of the sinusoidal perturbation (11) leads to the excitation of a larger frequency band in the solution $\widehat{u}_{h}(0.5, \xi)$, with $\nu_{\mathrm{f}}=3250, c=1280$, and $\nu_{\ell}=1$.

\section{$4 \quad$ Numerical examples}

In the following, the amplitude of emission is set to $A=1$, and the values of $J_{1}$ and $J_{2}$ (and thus the definition of $\mathcal{I}$ and $\mathcal{J}$, see $(22)$ ) are a priori selected relatively to the reference solution $\widehat{u}$ by the criterion

$$
\max _{j \in \mathbb{Z} \backslash \mathcal{I}}\left\|\widehat{u}_{h}\left(\cdot, \nu_{\mathrm{f}}+j \nu_{\ell}\right)\right\|_{\infty, \widetilde{\Omega}_{h}} \leq 10^{-3} \times\left\|\widehat{u}_{h}\left(\cdot, \nu_{\mathrm{f}}\right)\right\|_{\infty, \widetilde{\Omega}_{h}}
$$

in such a way that $\sharp \mathcal{I}^{+}$is minimized. This assumption ensures that $\widehat{u}_{h}^{\mathcal{J}^{+}}$is restricted to the significant components of $\widehat{u}_{h \nu_{\mathrm{f}}}$ around $\nu_{\mathrm{f}}$, with a normalized amplitude less than $10^{-3}$. Here, we set $\|f\|_{\infty, \widetilde{\Omega}_{h}}=\max _{\tilde{x} \in \widetilde{\Omega}_{h}}|f(\tilde{x})|$.

\subsection{Convergence of the frequency-domain solution $\widehat{w}_{\text {gen }, h}^{\mathcal{I}^{+}}$}

The computational cost of the proposed frequency domain method depend on the size $\sharp \mathcal{I}^{+}$of the system that we have to solve and on the number of finite elements $n_{\widetilde{\Omega}}=L / h$. The corresponding algorithm requires the solution of a linear system with a sparse block pentadiagonal matrix of size $n \sharp \mathcal{I}^{+}$, with tridiagonal blocks. On the other hand the brute force method used to compute the reference solution $\widehat{u}_{h}^{\mathcal{J}^{+}}$is based on the resolution of (29) with a $\mathbb{P}^{1}$ finite element method in space and a second-order Crank-Nicolson time scheme which requires the solution of $n_{t}$ tridiagonal linear systems of size $n_{\widetilde{\Omega}}$, followed by the computation of $n_{\widetilde{\Omega}}$ FFTs of size $n_{t}$.

In order to assess the accuracy of the method, we compare the numerical solutions $\widehat{w}_{\text {gen }, h}^{\mathcal{I}^{+}}$and $\widehat{u}_{h}^{\mathcal{J}^{+}}$in different physical settings. To this end, we first define the pointwise 
error between the Fourier spectra $\left|\widehat{u}_{h}^{\mathcal{J}^{+}}\right|$and $\left|\widehat{w}_{\text {gen }, h}^{\mathcal{I}^{+}}\right|$

$$
\operatorname{err}\left(\widehat{u}_{h}^{\mathcal{J}^{+}}, \widehat{w}_{\text {gen }, h}^{\mathcal{I}^{+}}, \widetilde{x}\right):=\frac{\left\|\left|\widehat{u}_{h}^{\mathcal{J}^{+}}(\widetilde{x}, \cdot)\right|-\left|\widehat{w}_{\text {gen }, h}^{\mathcal{I}^{+}}(\widetilde{x}, \cdot)\right|\right\|_{1, \mathcal{J}^{+}}}{\left\|\widehat{u}_{h}^{\mathcal{J}^{+}}(\widetilde{x}, \cdot)\right\|_{1, \mathcal{J}^{+}}}
$$

where $\|f\|_{1, \mathcal{J}^{+}}=\sum_{\xi \in \mathcal{J}^{+}}|f(\xi)|$.

Thanks to the non-homogeneous left boundary condition for the space-time problem (9), the denominator $\left\|\widehat{u}_{h}^{\mathcal{J}^{+}}(\widetilde{x}, \cdot)\right\|_{1, \mathcal{J}^{+}}$of $(33)$ never vanishes for $\widetilde{x} \in \widetilde{\Omega}_{h}$. Next, we define the overall error on the computational domain as

$$
\operatorname{Err}\left(\widehat{u}_{h}^{\mathcal{J}^{+}}, \widehat{w}_{\text {gen }, h}^{\mathcal{I}^{+}}\right):=\frac{1}{L} \int_{\widetilde{\Omega}_{h}} \operatorname{err}\left(\widehat{u}_{h}^{\mathcal{J}^{+}}, \widehat{w}_{\text {gen }, h}^{\mathcal{I}^{+}}, \widetilde{x}\right) d \widetilde{x}
$$

Figure $4 \mathrm{a}$ reports $\operatorname{Err}\left(\widehat{u}_{h}^{\mathcal{J}^{+}}, \widehat{w}_{\text {gen }, h}^{\mathcal{I}^{+}}\right)$with respect to increasing the source frequency $\nu_{\mathrm{f}}$, for different values of $h$. The time-domain solution $\widehat{u}_{h}^{\mathcal{J}^{+}}$was computed on the same spatial grid as $\widehat{w}_{\text {gen, } h}^{\mathcal{I}^{+}}$, with time discretization parameters adapted to reach the numerical convergence. In complement, we show in Figure $4 \mathrm{~b}$ the size of $\mathcal{I}^{+}$used to evaluate $\widehat{w}_{\text {gen }, h}^{\mathcal{I}^{+}}$vs. $\nu_{\mathrm{f}}$, with $c=300, \nu_{\ell}=1$ and $\epsilon=0.1$.

We observe that $\widehat{w}_{\text {gen }, h}^{\mathcal{I}^{+}}$provides a more accurate approximation of $\widehat{u}_{h}^{\mathcal{J}^{+}}$when the source frequency $\nu_{\mathrm{f}}$ is not close to an eigenfrequency $\nu_{m}$ of the system. Otherwise, the nearest eigenmode $\widehat{u}_{\nu_{m}}$ shares components of non negligible amplitude with $\widehat{u}_{\nu_{\mathrm{f}}}$, which makes the error increase. This sketches validity regions for the presented method, which correspond to the assumptions made in Section 3.3. Moreover, Figure $4 \mathrm{~b}$ illustrates that $\sharp \mathcal{I}^{+}$might stay relatively small on some regions of $\xi$ between the eigenfrequencies. In fact, the explicit determination of these precise regions is related to the a priori determination of $\sharp \mathcal{I}^{+}$and remains an open question.

However, it is very interesting to notice that for particular values of the ratio $\kappa_{\mathrm{f}}=$ $2 \pi \nu_{\mathrm{f}} / c$, the minimal size $\sharp \mathcal{I}^{+}$can remain small even for very large values of $\nu_{\mathrm{f}}$. For example, Figure 5 reports the spectrum $\left|\widehat{w}_{\mathrm{gen}, h}^{\mathcal{I}^{+}}\right|$for $\nu_{\mathrm{f}}=3.2 \times 10^{9}$ and $c=3 \times 10^{8}$, i.e. for a realistic radar application. In this case, 11 frequency components suffice for an accurate calculation of the solution.

\subsection{Influence of the number of frequency components $\sharp \mathcal{I}^{+}$}

Similarly to 34$)$, we report on Figure 6a the error $\operatorname{Err}\left(\widehat{u}_{h}^{\mathcal{J}^{+}}, \widehat{w}_{\text {gen }, h}^{\mathcal{I}^{+}}\right)$for different sizes $\sharp \mathcal{I}_{1}^{+}$ of the test interval $\mathcal{I}_{1}^{+}$. The reference interval $\mathcal{I}^{+}$is fixed with cardinal $\sharp \mathcal{I}^{+}=111$. In addition, Figure $6 \mathrm{~b}$ gives the error between $\widehat{w}_{\text {gen }, h}^{\mathcal{I}^{+}}$and $\widehat{w}_{\text {gen }, h}^{\mathcal{I}_{1}^{+}}$for $\sharp \mathcal{I}_{1}^{+}>111$. The parameters are $h=0.01, c=300, \nu_{\mathrm{f}}=500, \nu_{\ell}=1$ and $\epsilon=0.1$. The fast decay of the error is in accordance with the notion of minimal truncation interval $\sharp \mathcal{I}^{+}$, and $\operatorname{Err}\left(\widehat{u}_{h}^{\mathcal{J}^{+}}, \widehat{w}_{\text {gen }, h}^{\mathcal{I}_{1}^{+}}\right)$settles down about $3.5 \times 10^{-3}$ for $\sharp \mathcal{I}_{1}^{+} \geq 111$. In particular, for $\sharp \mathcal{I}_{1}^{+} \geq 111$, the triangular inequality entails that

$$
\operatorname{Err}\left(\widehat{u}_{h}^{\mathcal{J}^{+}}, \widehat{w}_{\text {gen }, h}^{\mathcal{I}_{1}^{+}}\right) \leq \operatorname{Err}\left(\widehat{u}_{h}^{\mathcal{J}^{+}}, \widehat{w}_{\text {gen }, h}^{\mathcal{I}^{+}}\right)+\operatorname{Err}\left(\widehat{w}_{\text {gen }, h}^{\mathcal{I}^{+}}, \widehat{w}_{\text {gen }, h}^{\mathcal{I}_{1}^{+}}\right) \approx 3.5 \times 10^{-3}+\operatorname{Err}\left(\widehat{w}_{\text {gen }, h}^{\mathcal{I}^{+}}, \widehat{w}_{\text {gen }, h}^{\mathcal{I}_{1}^{+}}\right),
$$




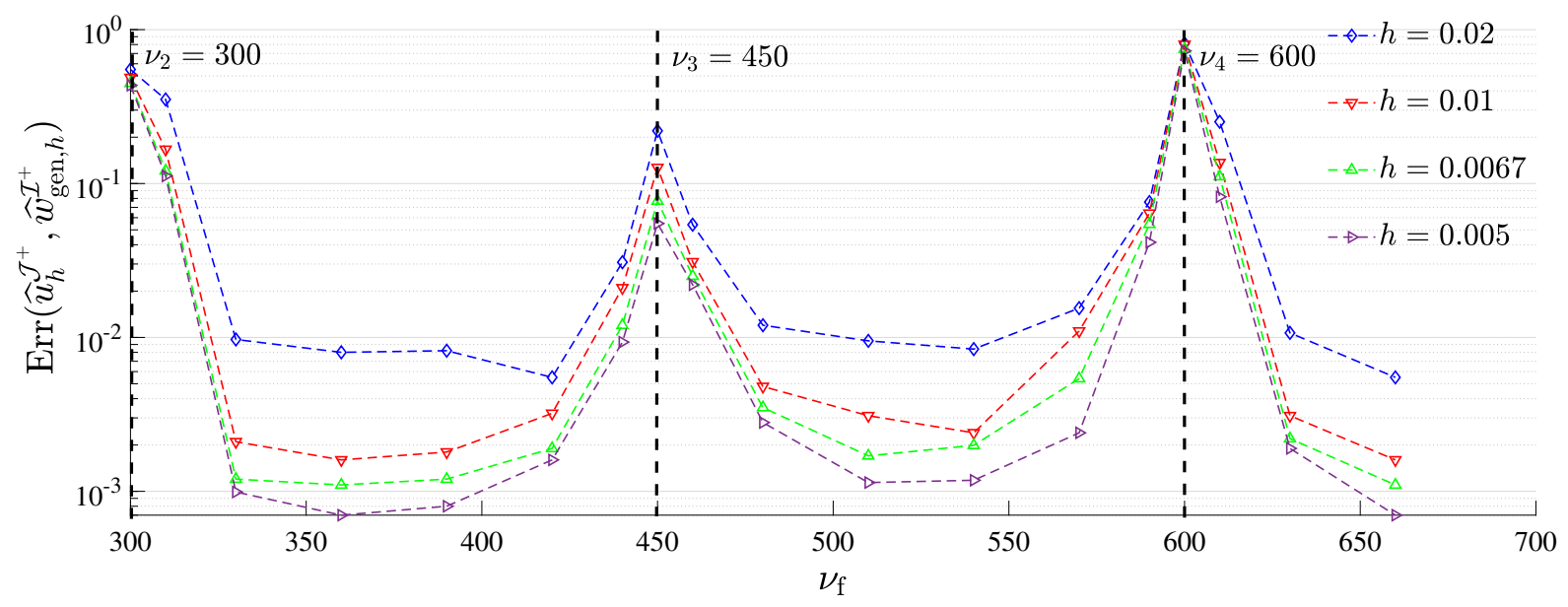

(a) $\operatorname{Err}\left(\widehat{u}_{h}^{\mathcal{J}^{+}}, \widehat{w}_{\text {gen }, h}^{\mathcal{I}^{+}}\right)$vs. $\nu_{\mathrm{f}}$ for decreasing $h$.

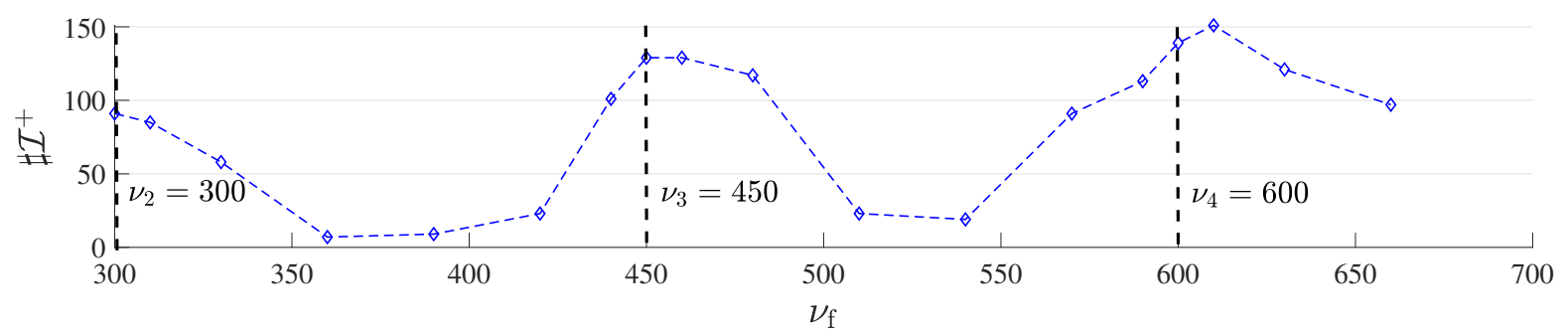

(b) $\sharp \mathcal{I}^{+}$vs. $\nu_{\mathrm{f}}$.

Fig. 4. Top: Error $\operatorname{Err}\left(\widehat{u}_{h}^{\mathcal{J}^{+}}, \widehat{w}_{\text {gen }, h}^{\mathcal{I}^{+}}\right)$depending on $\nu_{\mathrm{f}}$ for increasing refinement $h$. Bottom: cardinal $\sharp \mathcal{I}^{+}$vs. $\nu_{\mathrm{f}}$. The vertical dashed lines correspond to the eigenfrequencies $\nu_{m}=m c / 2$ (since $L=1$ ) of the spatial domain without boundary motion. 


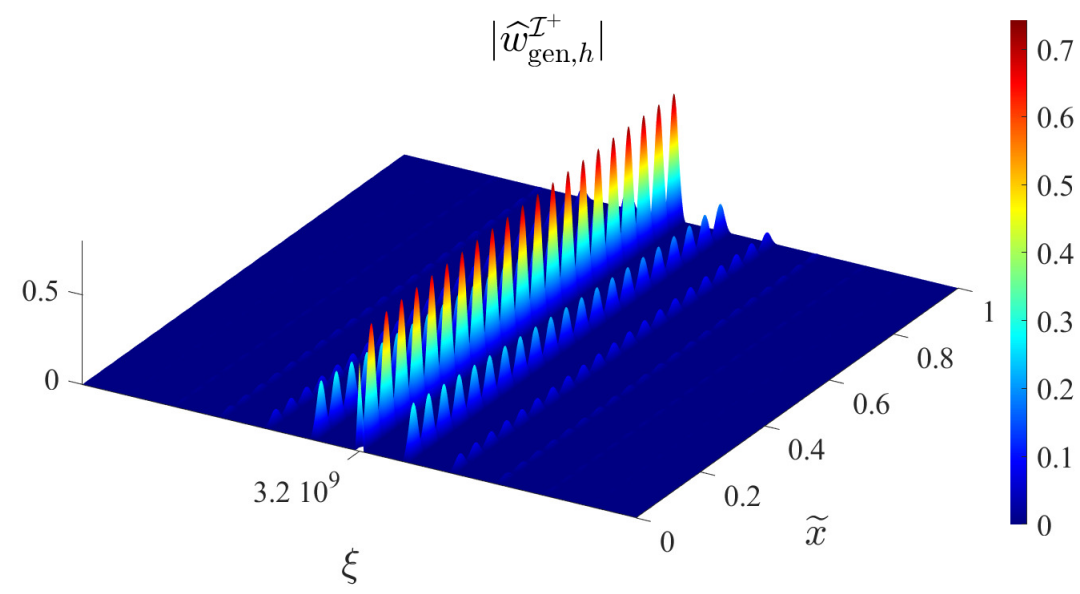

Fig. 5. Evaluation of $\left|\widehat{w}_{\text {gen }, h}^{\mathcal{I}^{+}}\right|$for a high frequency source, with $\nu_{\mathrm{f}}=3.2 \times 10^{9}$ and $c=3 \times 10^{8}$. The number of bumps along the space variable directly depends on the value of the wavenumber $\kappa_{\mathrm{f}}=2 \pi \nu_{\mathrm{f}} / c$. We use $\sharp \mathcal{I}^{+}=11, \epsilon=0.01, \nu_{\ell}=1$ and $h=0.001$.

and, as we observe in Figure $6 \mathrm{~b}$, this stabilization is of average amplitude $10^{-13}$. This illustrates the fast convergence of $\widehat{w}_{\text {gen, } h}^{\mathcal{I}^{+}}$with respect to the parameter $\sharp \mathcal{I}^{+}$. This result opens up the possibility to obtain a relevant estimation of $\mathcal{I}^{+}$, using iteratively the algorithm which computes $\widehat{w}_{\text {gen }, h}^{\mathcal{I}^{+}}$.

\subsection{Validity of the small-amplitude approximation $\widehat{w}_{h}^{\mathcal{I}^{+}}$}

To justify the range of validity of the proposed approximate solution $\widehat{w}^{\mathcal{I}^{+}}$, let us compare $\left|\widehat{w}_{h}^{\mathcal{I}^{+}}\right|$to $\left|\widehat{w}_{\text {gen }, h}^{\mathcal{I}^{+}}\right|$. In order to define $\mathcal{I}^{+}$for each physical configuration $\left\{c, \nu_{\mathrm{f}}, \nu_{\ell}, \epsilon\right\}$, we again use the criterion (32). Now, similarly to (34), we consider the error

$$
\operatorname{Err}\left(\widehat{w}_{\text {gen }, h}^{\mathcal{I}^{+}}, \widehat{w}_{h}^{\mathcal{I}^{+}}\right)=\frac{1}{L} \int_{\widetilde{\Omega}_{h}} \operatorname{err}\left(\widehat{w}_{\text {gen }, h}^{\mathcal{I}^{+}}, \widehat{w}_{h}^{\mathcal{I}^{+}}, \widetilde{x}\right) d \widetilde{x}
$$

In Figure 7, we report the error for increasing values of $\epsilon$ ranging from 0 to 0.2 and for $\nu_{\ell}$ in $[0,4]$. The other parameters are fixed to $\nu_{\mathrm{f}}=360$ and $c=300$. The spatial discretization step is $h=0.01$.

As expected, increasing the boundary motion amplitude $\epsilon$ and the frequency $\nu_{\ell}$ increases the relative difference between the two methods. Indeed, the dropped terms in (13) are becoming non negligible for relatively high magnitudes of $\epsilon$, which leads to define a region of validity of the approximation $\widehat{w}^{\mathcal{I}^{+}}$around small values of $\epsilon$. In particular, the choice of $\epsilon=0.1(1 / 10$ of the size of the domain $L=1)$ ensures a relative difference of $10^{-3}$ between $\widehat{w}_{h}^{\mathcal{I}^{+}}$and $\widehat{w}_{\text {gen }, h}^{\mathcal{I}^{+}}$.

In the following section, we provide a generalization of the frequency domain method to more complicated boundary motions, while maintaining $\epsilon \ll 1$. This assumption, in 


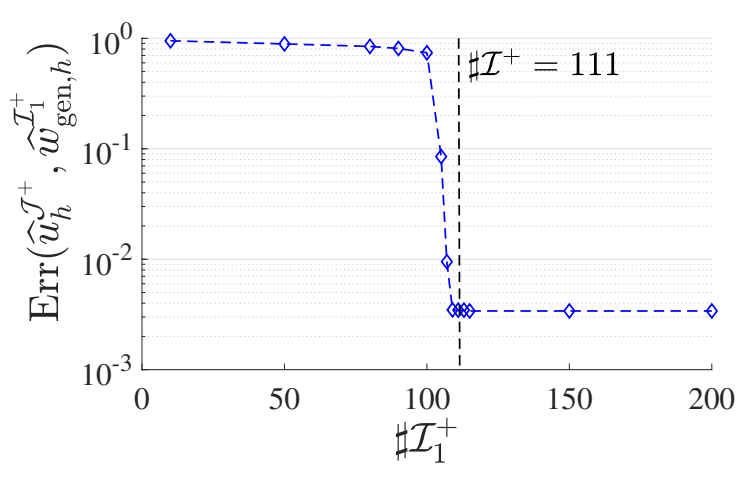

(a) $\operatorname{Err}\left(\widehat{u}_{h}^{\mathcal{J}^{+}}, \widehat{w}_{\text {gen }, h}^{\mathcal{I}_{1}^{+}}\right)$vs. $\sharp \mathcal{I}_{1}^{+}$

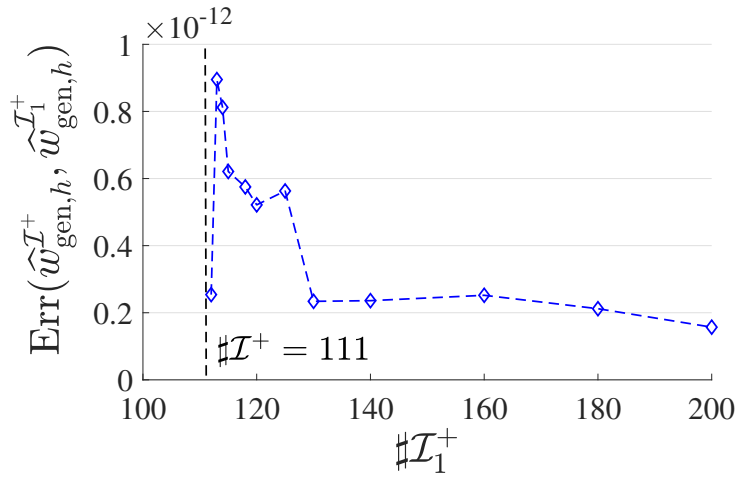

(b) $\operatorname{Err}\left(\widehat{w}_{\text {gen }, h}^{\mathcal{I}^{+}}, \widehat{w}_{\text {gen }, h}^{\mathcal{I}_{1}^{+}}\right)$vs. $\sharp \mathcal{I}_{1}^{+}$

Fig. 6. Left: error $\operatorname{Err}\left(\widehat{u}_{h}^{\mathcal{J}^{+}}, \widehat{w}_{\text {gen }, h}^{\mathcal{I}_{1}^{+}}\right)$depending on $\sharp \mathcal{I}_{1}^{+}$. Right: $\operatorname{Err}\left(\widehat{w}_{\text {gen }, h}^{\mathcal{I}^{+}}, \widehat{w}_{\text {gen }, h}^{\mathcal{I}_{1}^{+}}\right)$depending on $\sharp \mathcal{I}_{1}^{+}$. The dashed line corresponds to the fixed value $\sharp \mathcal{I}^{+}=111$. The parameters are $h=0.01$, $c=300, \nu_{\mathrm{f}}=500, \nu_{\ell}=1$ and $\epsilon=0.1$.

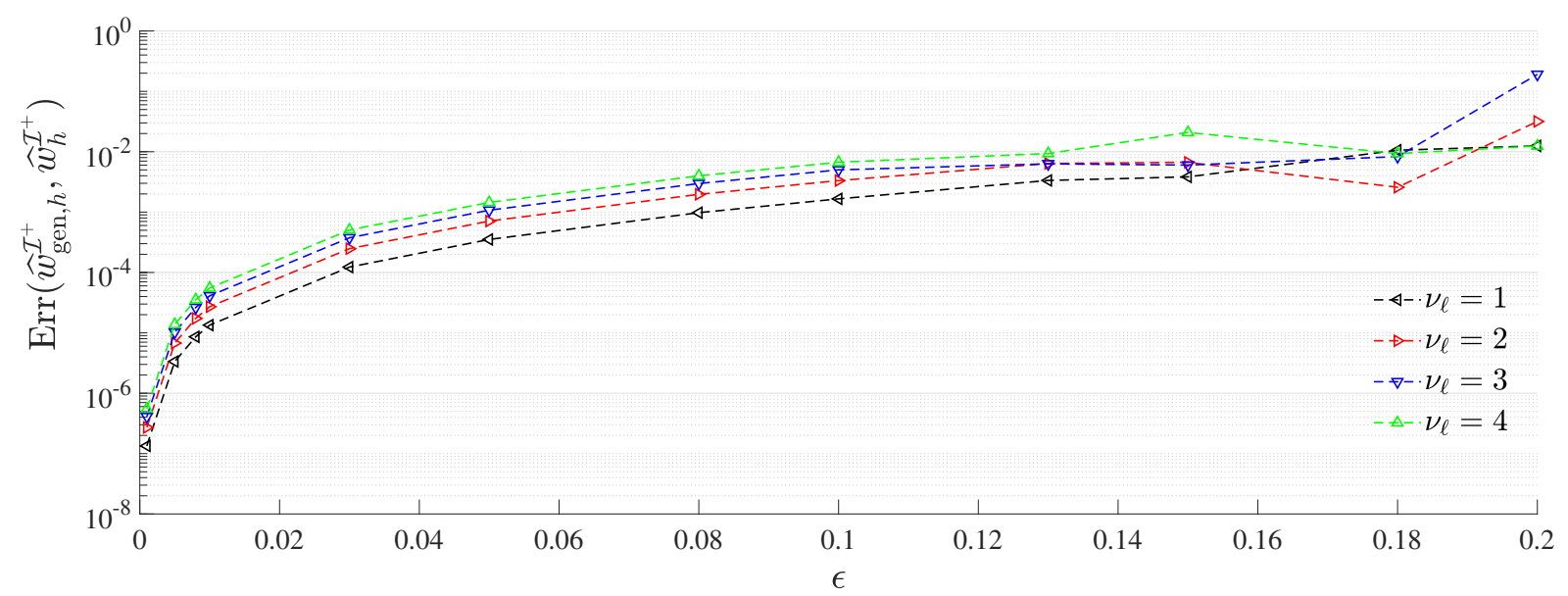

Fig. 7. $\operatorname{Err}\left(\widehat{w}_{\text {gen }, h}^{\mathcal{I}^{+}}, \widehat{w}_{h}^{\mathcal{I}^{+}}\right)$for increasing values of $\epsilon$ and $\nu_{\ell}$. 
accordance to the previous discussion and Figure 7, allows us to focus on developing the approximate numerical method $\widehat{w}^{\mathcal{I}^{+}}$for small $\epsilon$.

\section{$5 \quad$ Scattering with general boundary motions}

We introduce a generalization of the previous approach to a motion $\ell(t)$ which has no $a$ priori explicit expression. Let us denote by $\widetilde{u}(\widetilde{x}, t)$ the solution of $(9)$. We assume that the velocity of the motion of the boundary is much smaller than the phase speed of the emitted waves. In addition, we limit the study to the case where, for fixed $t$, the change of variables $\Omega(t)$ to $\widetilde{\Omega}$ is linear in spatial coordinates. The motion $\ell$ is expected to be a $\mathcal{C}^{\infty}(\mathbb{R})$ periodic time-dependent function taking its values in the interval $[L-\epsilon L, L+\epsilon L]$. Let us denote by $\nu_{\ell}$ the frequency of the motion.

Because $\ell$ never vanishes, Wiener $1 / f$ theorem [42] for Fourier series entails that the application $\widetilde{x}:(x, t) \mapsto \widetilde{x}(x, t)$ can be expanded as a time Fourier series around $\nu_{\ell}$. Then, by linearity of $x$ in $\widetilde{x}$ and due to the form of $\ell$, such an expansion also holds for $\left(\frac{\partial \tilde{x}}{\partial x}\right)^{2}$ and the corresponding time Fourier transform thus writes

$$
\mathcal{F}\left(\left(\frac{\partial \tilde{x}}{\partial x}\right)^{2}\right)=\sum_{n \in \mathbb{Z}} c_{n} \delta_{n \nu_{\ell}}
$$

for some complex-valued coefficients $c_{n}$. Since $\ell$ is not necessarily explicitly given, the computation of the coefficients $c_{n}$ of $\ell$ is based on the FFT in the time domain. Thanks to the linear of change of variable in $x$, the coefficients are constant in the spatial domain. Let us also notice that if the boundary motion is not periodic, the formulation of the Fourier transform is not a Dirac comb, which leads to a non-discrete system of equations. If it is almost-periodic in the sense of Bohr [3], a Fourier-type transform exists in the form $\sum_{n \in \mathbb{Z}} c_{n} \delta_{\lambda_{n}}$, with $c_{n} \in \mathbb{C}$ and $\lambda_{n} \in \mathbb{R}$. However, this expansion is based on the parameters $\lambda_{n}$ which are not necessarily multiples of $\lambda_{0}$. We do not treat these cases here.

We now consider (12), which approximates (9) in the case of small amplitude perturbations, but with a general motion $\ell$. The application of the Fourier transform on (12) yields

$$
\frac{4 \pi^{2} \xi^{2}}{c^{2}} \widehat{v}+\left(\sum_{n \in \mathbb{Z}} c_{n} \delta_{n \nu_{\ell}}\right) * \partial_{\tilde{x} \tilde{x}} \widehat{v}=0,
$$

with the boundary conditions $\widehat{v}(0, \xi)=0$ if $|\xi| \neq \nu_{\mathrm{f}}, \widehat{v}\left(0, \pm \nu_{\mathrm{f}}\right)= \pm \frac{A}{2 i}$, and $\widehat{v}(L, \xi)=0$ for all $\xi \in \mathbb{R}$. Since the boundary conditions are homogeneous with a harmonic source term, we assume that $\widetilde{v}$ admits a non-trivial expansion of the form (14). Then the arguments presented in Section 3 justify that we keep our focus on the component $\widehat{v}_{\nu_{\mathrm{f}}}$.

In the numerical simulations, the Fourier expansion (35) is truncated to $(2 N+1)$ terms. Indeed, since $\ell$ is $\mathcal{C}^{\infty}(\mathbb{R})$ and never vanishes, the Fourier coefficients $c_{n}$ are fastly decaying and we can therefore assume that the infinite sum (35) can be suitably truncated at order $N$ as $\sum_{n=-N}^{N} c_{n} \delta_{n \nu_{\ell}}$. Moreover, since $\ell$ is periodic with fundamental frequency $\nu_{\ell}$, it is 
reasonable to search for an approximate solution $\widehat{w}_{\nu_{\mathrm{f}}}^{\mathcal{I}^{+}}$of $\widehat{v}_{\nu_{\mathrm{f}}}$ under the form of the formal ansatz 26. Then, plugging the series expansion of $\widehat{w}_{\nu_{\mathrm{f}}}^{\mathcal{I}^{+}}$and 35 truncated at order $N$ into (36), and identifying the coefficients in front of the Dirac distributions leads to the following system of equations

$$
\kappa_{j}^{2} \widetilde{a}_{j}^{+}+\sum_{n=-N}^{N} c_{n} \partial_{\tilde{x} \tilde{x}} \widetilde{a}_{j-n}^{+}=0, \quad j \in \mathcal{I}^{+},
$$

where $J_{1}$ and $J_{2}$ have to be adequately chosen and $\kappa_{j}=\left(\omega_{\mathrm{f}}+j \omega_{\ell}\right) / c$. We have the boundary conditions: $\widetilde{a}_{0}^{+}(0)=\frac{A}{2 i}, \widetilde{a}_{j}^{+}(0)=0$ for $j \neq 0, \widetilde{a}_{j}^{+}(L)=0$ and $\widetilde{a}_{j}^{+}(\widetilde{x})=0$ for $j \in \mathbb{Z} \backslash \mathcal{I}^{+}$.

As previously, to solve (37) by the finite element method, we use the weak formulation: find $a_{j}^{*} \in \mathcal{H}_{\mathrm{D}_{j}}^{1}(\widetilde{\Omega})$ such that, for all $j \in \mathcal{I}^{+}$,

$$
\kappa_{j}^{2} \int_{\widetilde{\Omega}} a_{j}^{*} \phi d \widetilde{x}-\sum_{n=-N}^{N} c_{n} \int_{\widetilde{\Omega}} \partial_{\widetilde{x}} a_{j-n}^{*} \partial_{\widetilde{x}} \phi d \widetilde{x}=0,
$$

holds for all test functions $\phi \in \mathcal{H}_{0}^{1}(\widetilde{\Omega})$. The same covering $\widetilde{\Omega}_{h}$ of $\widetilde{\Omega}$ is used. The notation $\widehat{w}_{h}^{\mathcal{I}^{+}}$designates the solution of the discrete weak coupled system (38).

The case of a sine motion is already investigated in the previous sections. Let us consider the following function $\ell_{N_{\ell}}$ that involves $N_{\ell}$ modes

$$
\ell_{N_{\ell}}(t)=L\left(1+\epsilon \sum_{k=0}^{N_{\ell}} \frac{\sin \left((2 k+1) \omega_{\ell} t\right)}{2 k+1}\right) .
$$

For the numerical simulations, the interval $\mathcal{I}^{+}$is computed by using the previous criterion (32). For different values of $\epsilon \in[0.01,0.1]$, we report in Figure 8a the minimal cardinal $\sharp \mathcal{I}^{+}$ as a function of the number $N_{\ell}$ of modes in $\ell_{N_{\ell}}$. We keep the usual settings $A=1, c=300$, $\nu_{\mathrm{f}}=360, \nu_{\ell}=1$ and $h=0.01$. It is known that $\ell_{N_{\ell}}$ converges to the square shaped signal $\ell_{\infty}(t)=L+4 \operatorname{sign}(\sin (t))$ when $N_{\ell} \rightarrow+\infty$. As expected, the minimal cardinal $\sharp \mathcal{I}^{+}$of the system 38 increases with both $N_{\ell}$ and $\epsilon$. The error $\operatorname{Err}\left(\widehat{u}_{h}^{\mathcal{J}^{+}}, \widehat{w}_{h}^{\mathcal{I}^{+}}\right)$between the solution $\widehat{w}_{h}^{\mathcal{I}^{+}}$and the reference $\widehat{u}_{h}^{\mathcal{J}^{+}}$is reported in Figure $8 \mathrm{~b}$ for the same values of boundary motion amplitudes as in Figure 8a. The multi-harmonic formulation exhibits stable convergence w.r.t. the number of boundary modes, the error level being simply related to the small amplitude approximation used to compute $\widehat{w}_{h}^{\mathcal{I}^{+}}$.

\section{Extension to higher dimensions}

We now formally extend the approach developed for (1) to the space-time dimension $d+1$ (i.e. $d$ in space and 1 in time). To this end, we assume that a wave is emitted by a source $\Omega_{\mathrm{s}}$, with boundary $\Gamma_{\mathrm{s}}=\partial \Omega_{\mathrm{s}}$, and is scattered by an obstacle $\Omega_{\text {obst }}(t)$ with smooth boundary $\Gamma(t)=\partial \Omega_{\text {obst }}(t)$, moving with frequency $\nu_{\ell}$ around an equilibrium position $\Gamma(0)$. We then 


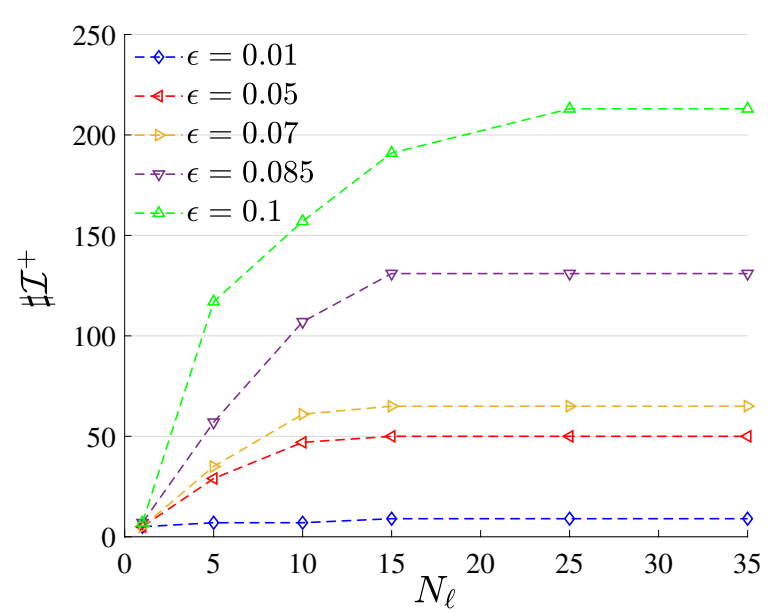

(a) $\sharp \mathcal{I}^{+}$vs. $N_{\ell}$.

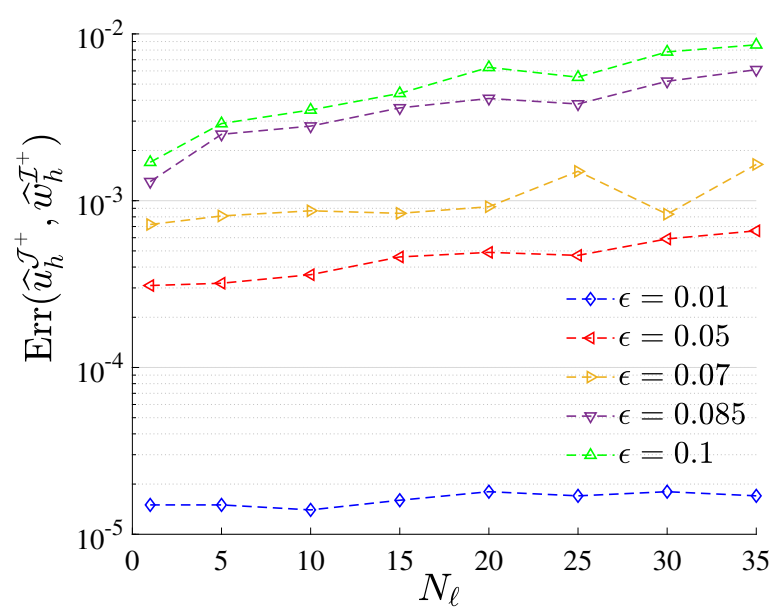

(b) $\operatorname{Err}\left(\widehat{u}_{h}^{\mathcal{J}^{+}}, \widehat{w}_{h}^{\mathcal{I}^{+}}\right)$vs. $N_{\ell}$.

Fig. 8. Left: $\sharp \mathcal{I}^{+}$vs. $N_{\ell}$. Right: error $\operatorname{Err}\left(\widehat{u}_{h}^{\mathcal{J}^{+}}, \widehat{w}_{h}^{\mathcal{I}^{+}}\right)$vs. $N_{\ell}$. The parameters are: $A=1$, $c=300, \nu_{\mathrm{f}}=360$, and $\nu_{\ell}=1$.

Fig. 9. Example of a two-dimensional domain $\Omega_{\text {ext }}(t)$ with source boundary $\Gamma_{\mathrm{s}}$ and moving scatterer boundary $\Gamma(t)$. 
define the $d$-dimensional domain of propagation, denoted by $\Omega_{\text {ext }}(t)$, as the exterior domain with boundaries $\Gamma_{\mathrm{s}}$ and $\Gamma(t)$. We schematically illustrate the configuration in Figure 9 ,

We define the position vector $\mathbf{r}:=\left(x_{1}, \ldots, x_{d}\right)^{T} \in \Omega_{\text {ext }}(t)$ and the associated Laplace operator $\Delta_{\mathbf{r}}:=\partial_{x_{1} x_{1}}+\ldots+\partial_{x_{d} x_{d}}$. Then, we obtain the extension of (1) as

$$
\partial_{t t} u-c^{2} \Delta_{\mathbf{r}} u=0
$$

with unknown total wave field $u(\mathbf{r}, t)$, for $\mathbf{r} \in \Omega_{\text {ext }}(t)$ and $t \in \mathbb{R}_{*}^{+}$. The field is given on $\Gamma_{\mathrm{s}}$ by $\left.u(\mathbf{r}, t)\right|_{\Gamma_{\mathrm{s}}}=A \sin \left(\omega_{\mathrm{f}} t\right)$. Since $\Omega_{\mathrm{ext}}(t)$ is unbounded, the system does not have any eigenmodes and the energy of the system is concentrated around the source frequency $\nu_{\mathrm{f}}$. The initial conditions are $u(\mathbf{r}, 0)=0$ and $\partial_{t} u(\mathbf{r}, 0)=0$.

Let us analyze the case of the three-dimensional spatial situation. We first map $\Omega_{\text {ext }}(t)$ to a fixed domain $\widetilde{\Omega}_{\text {ext }}$ with boundaries $\widetilde{\Gamma}_{\mathrm{s}}$ and $\widetilde{\Gamma}=\Gamma(0)$, by the change of space variable

$$
\widetilde{\mathbf{r}}:(\mathbf{r}, t) \mapsto \widetilde{\mathbf{r}}(\mathbf{r}, t)=\left(\widetilde{x}_{1}, \widetilde{x}_{2}, \widetilde{x}_{3}\right)^{T},
$$

such that $\widetilde{\mathbf{r}}(\Gamma(t), t)=\widetilde{\Gamma}$ and $\widetilde{\mathbf{r}}\left(\Gamma_{\mathrm{s}}, t\right)=\widetilde{\Gamma}_{\mathrm{s}}$. Then we set: $u(\mathbf{r}, t)=\widetilde{u}(\widetilde{\mathbf{r}}, t)$ for $t \in$ $\mathbb{R}_{*}^{+}$. For $\widetilde{\mathbf{x}}:=\left(\widetilde{x}_{1}, \widetilde{x}_{2}, \widetilde{x}_{3}, t\right)^{T} \in \widetilde{\Omega}_{\mathrm{ext}} \times \mathbb{R}_{*}^{+}$, let us define the gradient operators $\nabla_{\tilde{\mathbf{x}}}:=$ $\left(\partial_{\tilde{x}_{1}}, \partial_{\tilde{x}_{2}}, \partial_{\tilde{x}_{3}}, \partial_{t}\right)^{T}$ and $\nabla_{\tilde{\mathbf{r}}}:=\left(\partial_{\tilde{x}_{1}}, \partial_{\tilde{x}_{2}}, \partial_{\tilde{x}_{3}}\right)^{T}$. Then, we have $\partial_{t} u=\partial_{t}(\widetilde{u})=\partial_{t} \widetilde{\mathbf{x}} \cdot \nabla_{\tilde{\mathbf{x}}} \widetilde{u}$, and

$$
\begin{aligned}
\partial_{t t} u & =\partial_{t t} \widetilde{\mathbf{x}} \cdot \nabla_{\tilde{\mathbf{x}}} \widetilde{u}+\partial_{t} \widetilde{\mathbf{x}} \cdot \partial_{t}\left(\nabla_{\tilde{\mathbf{x}}} \widetilde{u}\right) \\
& =\partial_{t t} \widetilde{\mathbf{x}} \cdot \nabla_{\tilde{\mathbf{x}}} \widetilde{u}+\partial_{t} \widetilde{\mathbf{x}} \cdot \nabla_{\tilde{\mathbf{x}}} \partial_{t}(\widetilde{u}) \\
& =\partial_{t t} \widetilde{\mathbf{x}} \cdot \nabla_{\tilde{\mathbf{x}}} \widetilde{u}+\left(\partial_{t} \widetilde{\mathbf{x}} \cdot \nabla_{\tilde{\mathbf{x}}}\right)^{2} \widetilde{u}
\end{aligned}
$$

Let us define the time dependent spatial Jacobian matrix of $(\mathbf{r}, t) \mapsto \widetilde{\mathbf{r}}(\mathbf{r}, t)$ as $\mathbf{J}:=$ $\left[\partial_{x_{j}} \widetilde{x}_{i}\right]_{i, j \in\{1,2,3\}}$. Then, the spatial gradient writes $\nabla_{\mathbf{r}}=\mathbf{J}^{T} \nabla_{\tilde{\mathbf{r}}}$. Since $\nabla_{\mathbf{r}} t=0$, it directly follows that:

$$
\begin{aligned}
\Delta_{\mathbf{r}} u & =\nabla_{\mathbf{r}} \cdot \nabla_{\mathbf{r}} u \\
& =\nabla_{\mathbf{r}} \cdot\left(\mathbf{J}^{T} \nabla_{\tilde{\mathbf{r}}} \widetilde{u}\right) \\
& =\left(\nabla_{\mathbf{r}} \cdot \mathbf{J}^{T}\right) \nabla_{\tilde{\mathbf{r}}} \widetilde{u}+\nabla_{\tilde{\mathbf{r}}} \cdot \mathbf{J J}^{T} \nabla_{\tilde{\mathbf{r}} \widetilde{u}}
\end{aligned}
$$

Hence, substituting (40) and (41) into (39) leads to the equation

$$
\partial_{t t} \widetilde{\mathbf{x}} \cdot \nabla_{\tilde{\mathbf{x}}} \widetilde{u}+\left(\partial_{t} \widetilde{\mathbf{x}} \cdot \nabla_{\tilde{\mathbf{x}}}\right)^{2} \widetilde{u}-c^{2}\left[\left(\nabla_{\mathbf{r}} \cdot \mathbf{J}^{T}\right) \nabla_{\tilde{\mathbf{r}}} \widetilde{u}+\nabla_{\tilde{\mathbf{r}}} \cdot \mathbf{J} \mathbf{J}^{T} \nabla_{\tilde{\mathbf{r}}} \widetilde{u}\right]=0 .
$$

As previously, if we choose $\widetilde{x}_{i}, i=1,2,3$ of first order in $x_{i}$ we get $\left(\nabla_{\mathbf{r}} \cdot \mathbf{J}^{T}\right)=0$. Moreover if the amplitude of the movement is small and bounded by a small perturbation $\epsilon$, we can neglect the $\mathcal{O}(\epsilon)$ terms and the function $\partial_{t t} u$ in 40 reduces to $\partial_{t t} \widetilde{u}$. This leads to the following approximate equation for small amplitude boundary movements

$$
\partial_{t t} \widetilde{v}-c^{2} \nabla_{\tilde{\mathbf{r}}} \cdot \mathbf{J J}^{T} \nabla_{\tilde{\mathbf{r}}} \widetilde{v}=0
$$

which generalizes (12). In addition, we keep the previous homogeneous boundary condition on $\widetilde{\Gamma}$, the non-homogeneous boundary condition on $\widetilde{\Gamma}_{\mathrm{s}}$, i.e. $\left.\widetilde{v}(\widetilde{\mathbf{r}}, t)\right|_{\widetilde{\Gamma}_{\mathrm{s}}}=A \sin \left(\omega_{\mathrm{f}} t\right)$, and the 
initial conditions $\widetilde{v}(\widetilde{\mathbf{r}}, 0)=0$ and $\partial_{t} \widetilde{v}(\widetilde{\mathbf{r}}, 0)=0$. In practice, after discretization, we can choose a piecewise linear change of variable $\widetilde{\mathbf{r}}$, defined on the same mesh as the finite element solution, which makes $\mathbf{J}$ only time dependent on each finite element.

The Fourier transform in time of $(43)$ gives

$$
4 \pi^{2} \xi^{2} \widehat{v}+c^{2} \nabla_{\tilde{\mathbf{r}}} \cdot\left(\mathcal{F}\left(\mathbf{J J}^{T}\right) * \nabla_{\tilde{\mathbf{r}}} \widehat{v}\right)=0
$$

Following (37), we consider the truncated Fourier series expansion of $\mathbf{J J}^{T}(t)$

$$
\mathcal{F}\left(\mathbf{J J}^{T}\right)(\xi)=\sum_{n=-N}^{N} \mathbf{C}_{n} \delta_{n \nu_{\ell}}
$$

where $\mathbf{C}_{n}$ are some $3 \times 3$ complex-valued symmetric matrices. Proceeding as in Section 3.2 , we obtain from (44) the following system of coupled Helmholtz-type equations in terms of the complex Fourier coefficients $\widetilde{a}_{j}^{+}$, for $j \in \mathcal{I}^{+}$,

$$
\kappa_{j}^{2} \widetilde{a}_{j}^{+}+\nabla_{\tilde{\mathbf{r}}} \cdot \sum_{n=-N}^{N} \mathbf{C}_{n} \nabla_{\tilde{\mathbf{r}}} \widetilde{a}_{j-n}^{+}=0
$$

with $\kappa_{j}=\left(\omega_{\mathrm{f}}+j \omega_{\ell}\right) / c$. The boundary condition on $\widetilde{\Gamma}_{\mathrm{s}}$ can be written as $\widetilde{a}_{0}^{+}\left(\widetilde{\mathbf{r}}^{T}\right)=\frac{A}{2 i}$ and $\widetilde{a}_{j}^{+}\left(\widetilde{\mathbf{r}}^{T}\right)=0$ for $j \neq 0$. We apply the homogeneous Dirichlet boundary condition $\widetilde{a}_{j}^{+}\left(\widetilde{\mathbf{r}}^{T}\right)=0$ on $\widetilde{\Gamma}$ for $j \in \mathcal{I}^{+}$.

We now introduce a fictitious boundary $\widetilde{\Sigma}$ that delimits a finite computational domain $\widetilde{\Omega}$ with boundaries $\widetilde{\Sigma}, \widetilde{\Gamma}_{\mathrm{s}}$ and $\widetilde{\Gamma}$, and with exterior unit normal vector $\widetilde{\mathbf{n}}$. On $\widetilde{\Sigma}$, we impose the following absorbing boundary condition associated with each single Helmholtz equation:

$$
i \kappa_{j} \widetilde{a}_{j}^{+}+\widetilde{\mathbf{n}} \cdot \sum_{n=-N}^{N} \mathbf{C}_{n} \nabla_{\tilde{\mathbf{r}}} \widetilde{a}_{j-n}^{+}=0,
$$

for $j \in \mathcal{I}^{+}$. The derivation of this boundary condition is given in Appendix B.

We derive the following weak formulation of $(46):$ find $a_{j}^{*} \in \mathcal{H}_{\widetilde{\Gamma}_{\mathrm{s}}}^{1}(\widetilde{\Omega}):=\left\{a_{j}^{*} \in \mathcal{H}^{1}(\widetilde{\Omega}) \mid a_{0}^{*}(\widetilde{\mathbf{r}})=\right.$ $\frac{A}{2 i}$ and $a_{j}^{*}(\widetilde{\mathbf{r}})=0, j \neq 0$, on $\widetilde{\Gamma}_{\mathrm{s}}$, and $a_{j}^{*}(\widetilde{\mathbf{r}})=0$ on $\left.\widetilde{\Gamma}\right\}$ such that

$$
\kappa_{j}^{2} \int_{\widetilde{\Omega}} a_{j}^{*} \phi d \widetilde{\Omega}-\int_{\widetilde{\Omega}}\left(\sum_{n=-N}^{N} \mathbf{C}_{n} \nabla_{\tilde{\mathbf{r}}} a_{j-n}^{*}\right) \cdot \nabla_{\tilde{\mathbf{r}}} \phi d \widetilde{\Omega}-i \kappa_{j} \int_{\widetilde{\Sigma}} a_{j}^{*} \phi d \widetilde{\Sigma}=0,
$$

for all $\phi \in \mathcal{H}_{0, \widetilde{\Gamma}_{\mathrm{s}} \cup \widetilde{\Gamma}}^{1}(\widetilde{\Omega}):=\left\{\phi \in \mathcal{H}^{1}(\widetilde{\Omega}) \mid \phi=0\right.$ on $\left.\widetilde{\Gamma}_{\mathrm{s}} \cup \widetilde{\Gamma}\right\}$. We consider $\widetilde{\Omega}_{h}$ as a covering of $\widetilde{\Omega}$ consisting of $n_{\widetilde{\Omega}}$ triangular finite elements. For $j \in \mathcal{I}^{+}$, we denote by $a_{h, j}^{*}$ the linear finite element approximation of $a_{j}^{*}$.

We numerically illustrate the approach in the following two-dimensional $(d=2)$ model problem, where the initial domain is the rectangle $]-1 / 3, \ell(t)[\times]-1 / 2,1 / 2[$ where the right side of the rectangle $\Gamma(t)$ moves according to the sine motion $\ell(t)=1+\epsilon \sin \left(2 \pi \nu_{\ell} t\right)$. 


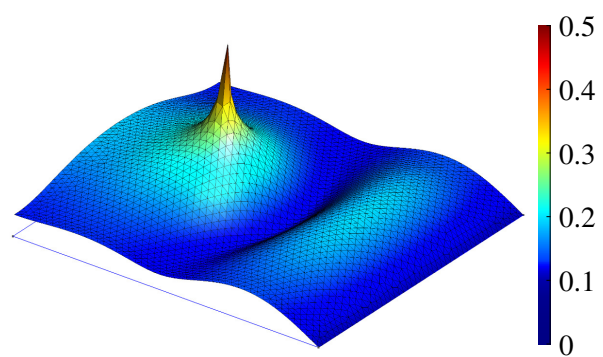

(a) $\left|a_{h, 0}^{*}(\widetilde{\mathbf{r}})\right|$

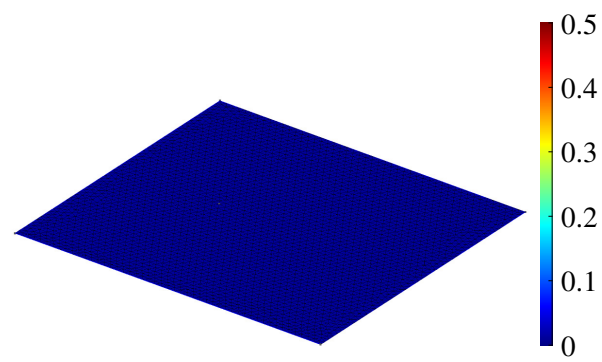

(c) $\left|a_{h, 2}^{*}(\widetilde{\mathbf{r}})\right|$

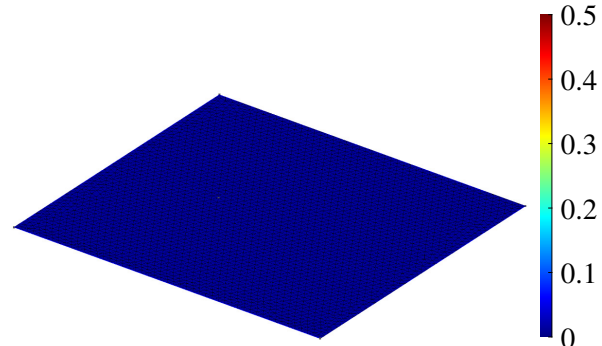

(b) $\left|a_{h, 1}^{*}(\widetilde{\mathbf{r}})\right|$

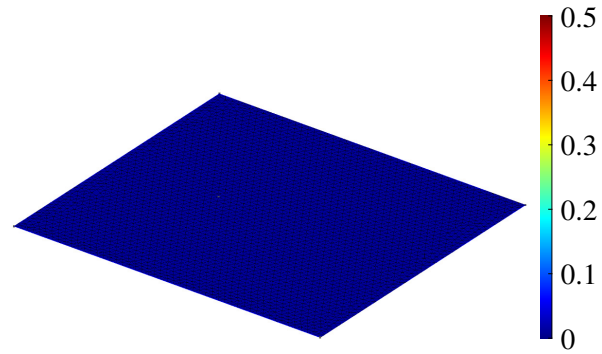

(d) $\left|a_{h, 3}^{*}(\widetilde{\mathbf{r}})\right|$

Fig. 10. Four components $\left|a_{j}^{*}(\widetilde{\mathbf{r}})\right|$ for $j=0,1,2,3$ of $\widehat{w}^{\mathcal{I}^{+}}(\widetilde{\mathbf{r}})$, for the physical parameters $\nu_{\mathrm{f}}=360$, $\nu_{\ell}=1, c=300$ and $\epsilon=0$. This case corresponds to the standard wave propagation in a fixed domain, without frequency modulation.

We consider absorbing boundary conditions on the three remaining sides and a point source $\Omega_{\mathrm{s}}$ located at $(0,0)^{T}$. The change of variable constitutes an extension of the onedimensional case (see Section 2.3), i.e. $\widetilde{\mathbf{r}}(\mathbf{r}, t)=\left(x_{1} / \ell(t), x_{2}\right)^{T}$. The discretized domain $\widetilde{\Omega}_{h}$ is a triangular mesh that consists of $n_{\widetilde{\Omega}}=640$ elements. In the Figures 10, 11 and 12, we report the amplitude of the four modes $\left|a_{h, 0}^{*}(\widetilde{\mathbf{r}})\right|,\left|a_{h, 1}^{*}(\widetilde{\mathbf{r}})\right|,\left|a_{h, 2}^{*}(\widetilde{\mathbf{r}})\right|$ and $\left|a_{h, 3}^{*}(\widetilde{\mathbf{r}})\right|$ of $\widehat{w}_{h}^{\mathcal{I}^{+}}(\widetilde{\mathbf{r}})$, for the configurations $\left(\nu_{\mathrm{f}}, \nu_{\ell}, c, \epsilon\right)=(360,1,300,0),(360,1,300,0.2)$ and $(3600,1,300,0.02)$, respectively. As previously, we keep $A=1$. As expected, in the case without perturbation, i.e. $\epsilon=0$ (see Figure 10p, only the mode $\left|a_{h, 0}^{*}(\widetilde{\mathbf{r}})\right|$ does not vanish, since there is no frequency modulation of the scattered wave. Contrary to the one-dimensional case, and since we compute the propagation of circular waves on a plane around the source $\widetilde{\mathbf{s}}$, the amplitudes $\left|a_{h, j}^{*}(\widetilde{\mathbf{r}})\right|$ of the modes of the field decay like $1 /\left|\widetilde{\mathbf{r}}-\widetilde{\Omega}_{\mathrm{s}}\right|$ as $\left|\widetilde{\mathbf{r}}-\widetilde{\Omega}_{\mathrm{s}}\right| \rightarrow \infty$. Moreover, in Figures 11 and $12,\left|a_{h, j}^{*}(\widetilde{\mathbf{r}})\right|$ decreases as $|j|$ increases, which is in line with the fast decay of the coefficients studied in Section 3.1. We also observe that, in accordance with the one-dimensional case, the spatial frequency of the solution $\left|a_{h, j}^{*}(\widetilde{\mathbf{r}})\right|$ increases with the wave number $\kappa_{j}$,

\section{Conclusion}

In this paper, we presented a new numerical method for solving the scattering problem of scalar waves by a moving $d$-dimensional obstacle with general movement. The method is 


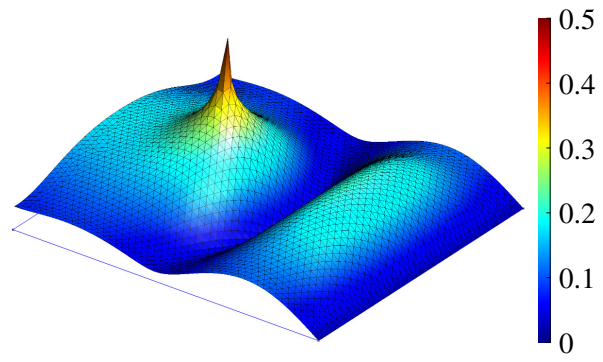

(a) $\left|a_{h, 0}^{*}(\widetilde{\mathbf{r}})\right|$

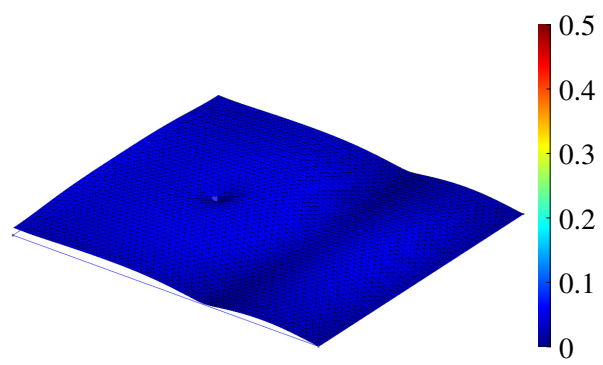

(c) $\left|a_{h, 2}^{*}(\widetilde{\mathbf{r}})\right|$

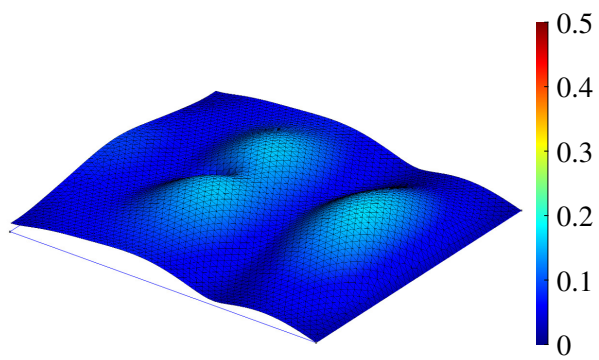

(b) $\left|a_{h, 1}^{*}(\widetilde{\mathbf{r}})\right|$

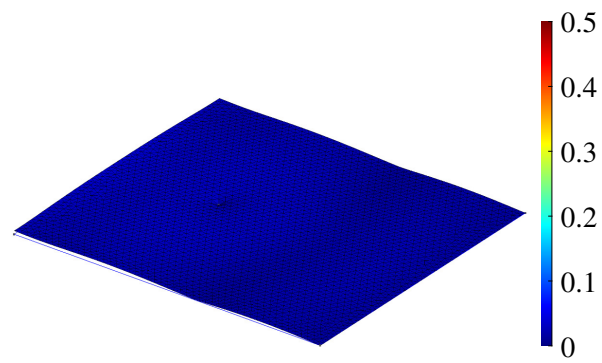

(d) $\left|a_{h, 3}^{*}(\widetilde{\mathbf{r}})\right|$

Fig. 11. Four components $\left|a_{j}^{*}(\widetilde{\mathbf{r}})\right|$ for $j=0,1,2,3$ of $\widehat{w}^{\mathcal{I}^{+}}(\widetilde{\mathbf{r}})$, for the physical parameters $\nu_{\mathrm{f}}=360$, $\nu_{\ell}=1, c=300$ and $\epsilon=0.2$.

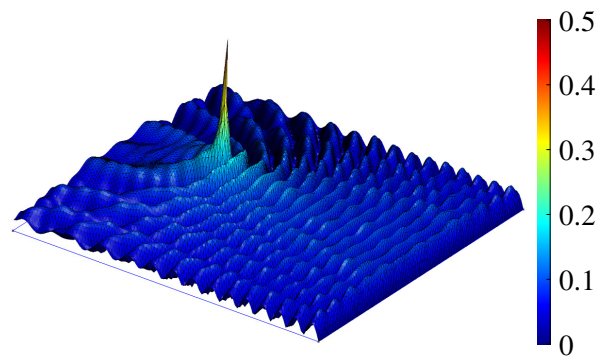

(a) $\left|a_{h, 0}^{*}(\widetilde{\mathbf{r}})\right|$

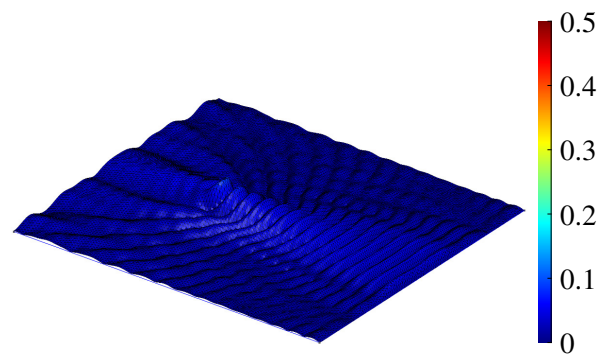

(c) $\left|a_{h, 2}^{*}(\widetilde{\mathbf{r}})\right|$

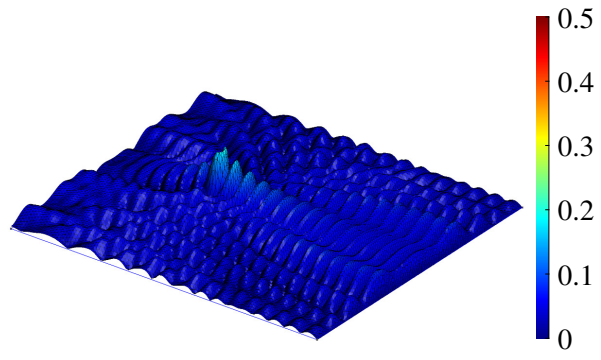

(b) $\left|a_{h, 1}^{*}(\widetilde{\mathbf{r}})\right|$

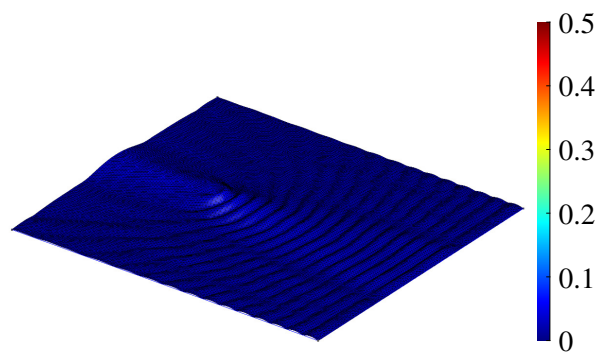

(d) $\left|a_{h, 3}^{*}(\widetilde{\mathbf{r}})\right|$

Fig. 12. Four components $\left|a_{j}^{*}(\widetilde{\mathbf{r}})\right|$ for $j=0,1,2,3$ of $\widehat{w}^{\mathcal{I}^{+}}(\widetilde{\mathbf{r}})$, for the physical parameters $\nu_{\mathrm{f}}=$ $3600, \nu_{\ell}=1, c=300$ and $\epsilon=0.02$. 
based on a change of variable which makes the moving domain fixed, and a multi-harmonic expansion of an approximate wave field. This results in the numerical solution of coupled systems of Helmholtz-type equations where optimized algorithms can be developed in the frequency domain. A preliminary numerical study is presented to confirm that the approach is accurate and efficient in the application domain of interest.

Ongoing works are now related to the design of optimized numerical algorithms for solving the coupled systems of harmonic equations and the extension to electromagnetic waves in view of industrial applications related to radar applications.

\section{A Proof of Proposition 1 .}

The source term of frequency $\nu_{\mathrm{f}}$ that continuously emits into the domain at the left boundary constitutes an initial impulse that excites the eigenmodes of the system at time $t=L / c$, which are solutions to the homogeneous wave equation (1) with zero boundary conditions.

To separate the different contributions in the global wave field, we decompose $u$ as the sum of the forced stationary wave coming from the source term and the free stationary waves related to the eigenmodes of the system. Hence, let us define

$$
u_{0}=u_{0, \nu_{\mathrm{f}}}+u_{0, \nu}
$$

where $u_{0, \nu_{\mathrm{f}}}(x, t)=E_{0, \nu_{\mathrm{f}}}(x) \sin \left(\omega_{\mathrm{f}} t\right)$, and $E_{0, \nu_{\mathrm{f}}}$ is the stationary solution to the Helmholtz equation with wavenumber $\kappa_{\mathrm{f}}:=\omega_{\mathrm{f}} / c$ in the bounded domain $] 0, L[$

$$
\left(\partial_{x x}+\kappa_{\mathrm{f}}^{2}\right) E_{0, \nu_{\mathrm{f}}}=0,
$$

with boundary conditions $E_{\nu_{\mathrm{f}}}(0)=A$ and $E_{\nu_{\mathrm{f}}}(L)=0$. A simple calculation gives $u_{0, \nu_{\mathrm{f}}}$ as

$$
u_{0, \nu_{\mathrm{f}}}(x, t)=\frac{A}{\sin \left(\kappa_{\mathrm{f}} L\right)} \sin \left(\kappa_{\mathrm{f}}(L-x)\right) \sin \left(\omega_{\mathrm{f}} t\right) .
$$

Let us now compute $u_{0, \nu}$. As a linear combination of stationary waves, the function $u_{0, \nu}$ is characterized by independent periodicities either in space and time, leading to the direct computation of the field $u_{0, \nu}$ by the separation of variables. More precisely, considering $u_{0, \nu}(x, t)=E_{0}(x) U_{0}(t)$ into (1), we obtain the following Helmholtz equation

$$
-\partial_{x x} E_{0}=\frac{\lambda^{2}}{c^{2}} E_{0}
$$

on $] 0, L[$, with homogeneous Dirichlet boundary conditions and for a constant $\lambda \in \mathbb{R}$. Since the spectrum of the Laplace operator in a bounded domain is discrete, the corresponding solutions for each eigenvalue $\lambda_{m}$ such that $\lambda_{m}:=m \pi c / L$ constitute an Hilbert basis $\left\{e_{\nu_{m}}\right\}_{m \in \mathbb{N}}$, with $e_{\nu_{m}}(x)=\sin \left(\frac{m \pi}{L} x\right)=: \sin \left(\kappa_{m} x\right)$ and $\omega_{m}:=2 \pi \nu_{m}=\kappa_{m} c=\frac{m \pi c}{L}$, for $m \in \mathbb{N}$. In addition, we have the ODE

$$
-\partial_{t t} U_{0}=\omega_{m}^{2} U_{0}
$$


for $m \in \mathbb{N}$, with $U(0)=0$. For each frequency $\nu_{m}$, the solution is then of the form

$$
u_{0, \nu_{m}}(t)=\phi_{m} \sin \left(\omega_{m}(t+\psi)\right) \text {, }
$$

where $\phi_{m} \in \mathbb{R}$, for $m \in \mathbb{N}$, and $\psi$ must be determined. Since $u_{0, \nu_{\mathrm{f}}}(x, 0)=0$, (4) and (49) imply that $u_{0, \nu_{m}}(x, 0)=0$ and then $\psi=0$. Hence, by the superposition principle, the general solution $u_{0, \nu}$ for the free modes can then be written as

$$
u_{0, \nu}(x, t)=\sum_{m \in \mathbb{N}} u_{0, \nu_{m}}(t) e_{\nu_{m}}(x)=\sum_{m \in \mathbb{N}} \phi_{m} \sin \left(\omega_{m} t\right) e_{\nu_{m}}(x),
$$

which entails that

$$
\partial_{t} u_{0, \nu}(x, 0)=\sum_{m \in \mathbb{N}} \phi_{m} \omega_{m} e_{\nu_{m}}(x) .
$$

Since each stationary mode is $\mathcal{C}^{\infty}\left(\Omega(t) \times \mathbb{R}_{*}^{+}\right)$, the convergence of the sum is guaranteed. Moreover, (5) and (49) lead to

$$
\partial_{t} u_{0, \nu_{m}}(x, 0)=-\partial_{t} u_{0, \nu_{\mathrm{f}}}(x, 0) .
$$

Hence, using (50) and (52), one gets by a direct computation

$$
\partial_{t} u_{0, \nu}(x, 0)=-\frac{A \omega_{\mathrm{f}}}{\sin \left(\kappa_{\mathrm{f}} L\right)} \sin \left(\kappa_{\mathrm{f}}(L-x)\right) .
$$

Let us consider the $L$-periodic odd extension of $\sin \left(\kappa_{\mathrm{f}}(L-x)\right)$ in (53). Expanding it in the basis $\left\{e_{\nu_{m}}\right\}_{m \in \mathbb{N}}$ for $\nu_{0, \mathrm{f}} \neq \nu_{m}$, we obtain for $\left.x \in\right] 0, L[$

$$
\sin \left(\kappa_{\mathrm{f}}(L-x)\right)=\sum_{m \in \mathbb{N}} \varphi_{m} e_{\nu_{m}}(x)=\sum_{m \in \mathbb{N}} \varphi_{m} \sin \left(\kappa_{m} x\right)
$$

where it is well-known that the odd Fourier coefficient $\varphi_{m}$ satisfies

$$
\begin{aligned}
\varphi_{m} & =\frac{2}{L} \int_{0}^{L} \sin \left(\kappa_{\mathrm{f}}(L-x)\right) \sin \left(\kappa_{m} x\right) d x \\
& =\frac{1}{L} \int_{0}^{L}\left[\cos \left(x\left(\kappa_{m}+\kappa_{\mathrm{f}}\right)-\kappa_{\mathrm{f}} L\right)-\cos \left(x\left(\kappa_{m}-\kappa_{\mathrm{f}}\right)+\kappa_{\mathrm{f}} L\right)\right] d x \\
& =\frac{2 \kappa_{m} \sin \left(\kappa_{\mathrm{f}} L\right)}{L\left(\kappa_{m}^{2}-\kappa_{\mathrm{f}}^{2}\right)}
\end{aligned}
$$

Finally, by identification with (51), we conclude that

$$
\phi_{m}=\frac{2 A \kappa_{\mathrm{f}}}{L\left(\kappa_{\mathrm{f}}^{2}-\kappa_{m}^{2}\right)} .
$$

Finally, for $\nu_{\mathrm{f}} \neq \nu_{m}$, we obtain the result by replacing $u_{0, \nu_{\mathrm{f}}}$ and $u_{0, k \nu}$ in 49 . 


\section{B Derivation of the absorbing boundary condition (47)}

Let us consider the lower-order absorbing boundary condition applied to the outer boundary of $\Omega_{\text {ext }}(t)$

$$
\frac{1}{c} \partial_{t} u+\mathbf{n} \cdot \nabla_{\mathbf{r}} u=0
$$

with outwardly directed unit normal vector $\mathbf{n}$. The deformation field $\widetilde{\mathbf{r}}$ implies that the corresponding unit normal vector $\widetilde{\mathbf{n}}$ to $\widetilde{\Sigma}$ satisfies: $\mathbf{J}^{T} \widetilde{\mathbf{n}}=\mathbf{n}$. Hence, considering the assumptions that lead to derive (43), we have the following equivalent absorbing boundary condition on $\widetilde{\Sigma}$

$$
\frac{1}{c} \partial_{t} \widetilde{v}+\widetilde{\mathbf{n}} \cdot \mathbf{J} \mathbf{J}^{T} \nabla_{\tilde{\mathbf{r}}} \widetilde{v}=0 .
$$

The time Fourier transform of (57) leads to

$$
\frac{2 i \pi \xi}{c} \widehat{v}+\widetilde{\mathbf{n}} \cdot \mathcal{F}\left(\mathbf{J J}^{T}\right) * \nabla_{\tilde{\mathbf{r}}} \widehat{v}=0 .
$$

Plugging the Fourier series expansion (45) of $\mathbf{J J}^{T}(t)$ into (58), we obtain the following system of absorbing boundary conditions in terms of the complex Fourier coefficients $\widetilde{a}_{j}$, for $j \in \mathcal{I}$ :

$$
i \kappa_{j} \widetilde{a}_{j}+\widetilde{\mathbf{n}} \cdot \sum_{n=-N}^{N} \mathbf{C}_{n} \nabla_{\tilde{\mathbf{r}}} \widetilde{a}_{j-n}=0,
$$

with $\kappa_{j}=\left(\omega_{\mathrm{f}}+j \omega_{\ell}\right) / c$.

\section{Acknowledgements.}

The authors thank the support of the Luxembourg National Research Fund (FNR) (2017-1 PPP 11608832). This research was funded in part through the ARC grant for Concerted Research Actions (ARC WAVES 15/19-03), financed by the Wallonia-Brussels Federation of Belgium.

\section{References}

[1] M. Abramowitz and I.A. Stegun. Handbook of Mathematical Functions, with Formulas, Graphs, and Mathematical Tables. Dover Publications, 1964.

[2] V. Agnihotri, M. Sabharwal, and V. Goyal. Effect of frequency on micro-Doppler signatures of a helicopter. In 2019 International Conference on Advances in Big Data, Computing and Data Communication Systems (icABCD), pages 1-5, 082019.

[3] H. Bohr. Almost Periodic Functions. American Mathematical Society, 1947.

[4] Y. Boubendir, X. Antoine, and C. Geuzaine. A quasi-optimal non-overlapping domain decomposition algorithm for the Helmholtz equation. Journal of Computational Physics, 231(2):262-280, 2012. 
[5] D. Bouche, D. Molinet, and R. Mittra. Asymptotic Methods in Electromagnetism. Springer, 1997.

[6] H.G. Brachtendorf, G. Welsch, and R. Laur. Fast simulation of the steady-state of circuits by the harmonic balance technique. In Proceedings of ISCAS'95 - International Symposium on Circuits and Systems, volume 2, pages 1388-1391, 1995.

[7] D. Brooks, O. Schwander, F. Barbaresco, J.-Y. Schneider, and M. Cord. Complexvalued neural networks for fully-temporal micro-Doppler classification. In 20th International Radar Symposium (IRS), pages 1-10, 2019.

[8] D.A. Brooks, O. Schwander, F. Barbaresco, J.-Y. Schneider, and M. Cord. Temporal deep learning for drone micro-Doppler classification. In Rohling, H, editor, 2018 19th International Radar Symposium (IRS), 2018.

[9] Z. A. Cammenga, C. J. Baker, G. E. Smith, and R. Ewing. Micro-Doppler target scattering. In IEEE Radar Conference, pages 1451-1455, 2014.

[10] Z.A. Cammenga, G.E. Smith, and C.J. Baker. High range resolution micro-Doppler analysis. In Ranney, KI and Doerry, A and Gilbreath, GC and Hawley, CT, editor, Radar Sensor Technology XIX; and Active and Passive Signatures VI, volume 9461 of Proceedings of SPIE, 2015.

[11] A. Cardona, T. Coune, A. Lerusse, and M. Geradin. A multiharmonic method for nonlinear vibration analysis. International Journal for Numerical Methods in Engineering, 37(9):1593-1608, 1994.

[12] D. Censor. Scattering of electromagnetic waves by a cylinder moving along its axis. IEEE Transactions on Microwave Theory and Techniques, 17(3):154-158, 1969.

[13] D. Censor. Scattering of electromagnetic waves in uniformly moving media. Journal of Mathematical Physics, 11(6):1968-1976, 1970.

[14] D. Censor. Non-relativistic scattering: pulsating interfaces. Progress In Electromagnetics Research, 54:263-281, 2005.

[15] V.C. Chen. The Micro-Doppler Effect in Radar, 2nd Ed. Artech House, 2 edition, 2019.

[16] V.C. Chen, F.Y. Li, S.S. Ho, and H. Wechsler. Micro-Doppler effect in radar: phenomenon, model, and simulation study. IEEE Transactions on Aerospace and Electronic Systems, 42(1):2-21, 2006.

[17] V.C. Chen, C.-T. Lin, and W.P. Pala. Time-varying Doppler analysis of electromagnetic backscattering from rotating object. In 2006 IEEE Radar Conference, Vols 1 and 2, IEEE Radar Conference, pages 807+, 2006. 
[18] X. Chen, X. Yu, J. Guan, and Y. He. High-resolution sparse representation of microDoppler signal in sparse fractional domain, volume 227 of Lecture Notes of the Institute for Computer Sciences, Social Informatics and Telecommunications Engineering, pages 225-232. 2018.

[19] W.C. Chew, E. Michielssen, J.M. Song, and J.M Jin. Fast and Efficient Algorithms in Computational Electromagnetics. Artech House, Inc., 2001.

[20] I.C. Christov and C.I. Christov. On mechanical waves and Doppler shifts from moving boundaries. Mathematical Methods in the Applied Sciences, 40(12):4481-4492, 2017.

[21] C.W. Chuang. Backscatter of a large rotating conducting cylinder of arbitrary crosssection. IEEE Transactions on Antennas and Propagation, 27(1):92-95, 1979.

[22] B. Cochelin and C. Vergez. A high order purely frequency-based harmonic balance formulation for continuation of periodic solutions. Journal of Sound and Vibration, 324(1-2):243-262, 2009.

[23] S. Dias Da Cruz, H-P. Beise, U. Schröder, and U. Karahasanovic. A theoretical investigation of the detection of vital signs in presence of car vibrations and radar-based passenger classification. IEEE Transactions on Vehicular Technology, 68(4):33743385, 2019.

[24] V. Dolean, P. Jolivet, and F. Nataf. An Introduction to Domain Decomposition Methods: Theory and Parallel Implementation. SIAM, Philadelphia, 2015.

[25] P. Dular, C. Geuzaine, F. Henrotte, and N. Legros. A general environment for the treatment of discrete problems and its application to the finite element method. IEEE Transactions on Magnetics, 34(5, 1):3395-3398, 1998.

[26] J.F. Dunne and P. Hayward. A split-frequency harmonic balance method for nonlinear oscillators with multi-harmonic forcing. Journal of Sound and Vibration, 295(3-5):939963, 2006.

[27] M. El Bouajaji, B. Thierry, X. Antoine, and C. Geuzaine. A quasi-optimal domain decomposition algorithm for the time-harmonic Maxwell's equations. Journal of Computational Physics, 294:38-57, 2015.

[28] A.S. Fokas and B. Pelloni. Method for solving moving boundary value problems for linear evolution equations. Physical Review Letters, 84(21):4785-4789, 2000.

[29] J. Garcia-Rubia, O. Kilic, V. Dang, Q. Nguyen, and T. Nghia. Analysis of moving human micro-Doppler signature in forest environments. Progress In Electromagnetics Research, 148:1-14, 062014.

[30] T.P. Gill. The Doppler Effect: An Introduction to the Theory of the Effect. Logos Press, 1965. 
[31] C. Gu, G. Wang, Y. Li, T. Inoue, and C. Li. A hybrid radar-camera sensing system with phase compensation for random body movement cancellation in Doppler vital sign detection. IEEE Transactions on Microwave Theory and Techniques, 61(12):4678-4688, 2013.

[32] J. Gyselinck, C. Geuzaine, P. Dular, and W. Legros. Multi-harmonic modelling of motional magnetic field problems using a hybrid finite element-boundary element discretisation. Journal of Computational and Applied Mathematics, 168(1-2):225-234, 2004.

[33] A. Halbach and C. Geuzaine. Steady-state nonlinear analysis of large arrays of electrically actuated micromembranes vibrating in a fluid. Engineering with Computers, 155(3):591-602, 2017.

[34] F. Harfoush, A. Taflove, and G.A. Kriegsmann. A numerical technique for analyzing electromagnetic wave scattering from moving surfaces in one and two dimensions. IEEE Transactions on Antennas and Propagation, 37(1):55-63, 1989.

[35] P. Ju. Global residue harmonic balance method for Helmholtz-Duffing oscillator. Applied Mathematical Modelling, 39(8):2172-2179, 2015.

[36] U. Karahasanovic and D. Tatarinov. Radar-based detection of thoracoabdominal asynchrony during breathing using autocorrelation function analysis. In 2018 11th German Microwave Conference (GEMIC 2018), pages 403-406, 2018.

[37] P. Kuchment. Floquet Theory For Partial Differential Equations. Birkhauser Verlag, Basel, 1993.

[38] C. Li, J. Cummings, J. Lam, E. Graves, and W. Wu. Radar remote monitoring of vital signs. Microwave Magazine, IEEE, 10:47-56, 2009.

[39] Z. Liu, B. Peng, and X. Li. Analysis of phase noise influence on micro-Doppler feature extraction of vibrating target. Journal of Engineering-JOE, 2019(20):6834-6839, OCT 2019.

[40] Z. Liu, B. Peng, and Li X. Analysis of phase noise influence on micro-Doppler feature extraction on vibrating target. Progress in Electromagnetics Research C, 85:177-190, 2018.

[41] R. Mickens. A generalization of the method of harmonic balance. Journal of Sound and Vibration, 111(3):515-518, 1986.

[42] D.J. Newman. A simple proof of Wiener $1 / f$ theorem. Proceedings of the American Mathematical Society, 48(1):264-265, 1975. 
[43] B. Peng, X. Wei, B. Deng, H. Chen, Z. Liu, and X. Li. A sinusoidal frequency modulation Fourier transform for radar-based vehicle vibration estimation. IEEE Transactions on Instrumentation and Measurement, 63(9):2188-2199, 2014.

[44] B. Thierry, A. Vion, S. Tournier, M. El Bouajaji, D. Colignon, N. Marsic, X. Antoine, and C. Geuzaine. GetDDM: An open framework for testing optimized Schwarz methods for time-harmonic wave problems. Computer Physics Communications, 203:309330, 2016.

[45] J. Van Bladel. Electromagnetic fields in the presence of rotating bodies. Proceedings of the IEEE, 64(3):301-318, 1976.

[46] C.W. Wong, W.S. Zhang, and S.L. Lau. Periodic forced vibration of unsymmetrical piecewise-linear systems by incremental harmonic-balance method. Journal of Sound and Vibration, 149(1):91-105, 1991.

[47] S. Yamada and K. Bessho. Harmonic field calculation by the combination of finite element analysis and harmonic-balance method. IEEE Transactions on Magnetics, 24(6):2588-2590, 1988.

[48] H. L. Zhang, Y. X. Sha, X. Y. Guo, M. Y. Xia, and C. H. Chan. Efficient analysis of scattering by multiple moving objects using a tailored MLFMA. IEEE Transactions on Antennas and Propagation, 67(3):2023-2027, 2019.

[49] K. Zheng, Y. Li, S. Qin, K. An, and G. Wei. Analysis of micromotion characteristics from moving conical-shaped targets using the Lorentz-FDTD method. IEEE Transactions on Antennas and Propagation, 67(11):7174-7179, 2019.

[50] K.-S. Zheng, J. Z. Li, G. Wei, and J.-D. Xu. Analysis of Doppler effect of moving conducting surfaces with Lorentz-FDTD method. Journal of Electromagnetic Waves and Applications, 27(2):149-159, 2013. 